\title{
Constraints on the gluon PDF from top quark pair production at hadron colliders
}

\section{Michal Czakon, ${ }^{a}$ Michelangelo L. Mangano, ${ }^{b}$ Alexander Mitov ${ }^{b}$ and Juan Rojo $^{b}$}

\author{
${ }^{a}$ Institut fur Theoretische Teilchenphysik und Kosmologie, \\ RWTH Aachen University, D-52056 Aachen, Germany \\ ${ }^{b}$ Physics Department, Theory Unit, CERN, \\ CH-1211 Genève, Switzerland \\ E-mail: mczakon@physik.rwth-aachen.de, michelangelo.mangano@cern.ch, \\ alexander.mitov@cern.ch, juan.rojo@cern.ch
}

ABSTRACT: Using the recently derived NNLO cross sections [1], we provide NNLO+NNLL theoretical predictions for top quark pair production based on all the available NNLO PDF sets, and compare them with the most precise LHC and Tevatron data. In this comparison we study in detail the PDF uncertainty and the scale, $m_{t}$ and $\alpha_{s}$ dependence of the theoretical predictions for each PDF set. Next, we observe that top quark pair production provides a powerful direct constraint on the gluon PDF at large $x$, and include Tevatron and LHC top pair data consistently into a global NNLO PDF fit. We then explore the phenomenological consequences of the reduced gluon PDF uncertainties, by showing how they can improve predictions for Beyond the Standard Model processes at the LHC. Finally, we update to full NNLO+NNLL the theoretical predictions for the ratio of top quark cross sections between different LHC center of mass energies, as well as the cross sections for hypothetical heavy fourth-generation quark production at the LHC.

KeYwords: QCD Phenomenology, Hadronic Colliders

ARXiv EPRINT: 1303.7215 


\section{Contents}

1 Introduction $\quad 1$

2 Settings 3

3 The top cross-section: measurements, predictions and uncertainties 4

3.1 Comparison between the various PDF sets 5

3.2 Uncertainty due to the value of $m_{t} \quad 8$

$\begin{array}{lll}3.3 & \text { Uncertainty due to the value of } \alpha_{s} & 9\end{array}$

4 Top quark data constraints on the gluon PDF 10

$\begin{array}{ll}4.1 & \text { Impact on predictions for BSM particle production } \\ \end{array}$

5 Cross-section ratios between different LHC beam energies 16

6 Hypothetical fourth-generation heavy quark production at the LHC $\quad 19$

$\begin{array}{lll}7 & \text { Summary and outlook } & 20\end{array}$

\section{Introduction}

The total $t \bar{t}$ production cross section is an important observable at hadron colliders, which has been recently computed in full next-to-next-to leading order (NNLO) QCD [1-4]. Thanks to a much improved control over higher order terms, including soft gluon emissions through next-to-next-to leading log (NNLL) [5-7], scale uncertainties are now controlled down to the 2.2 (3) percent level at the Tevatron (LHC), enabling a number of precision phenomenology applications to SM and BSM physics.

In this work we present an in-depth study of the theoretical uncertainties that affect the total cross section computed with NNLO+NNLL precision. These uncertainties are the parton distributions of the proton, the value of the strong coupling $\alpha_{s}\left(M_{Z}\right)$, the value of the top quark mass $m_{t}$ and the scale uncertainties from missing higher perturbative orders. We then compare the theoretical predictions with the most precise available data from the Tevatron and the LHC at 7 and $8 \mathrm{TeV}$. We also provide predictions for LHC at $14 \mathrm{TeV}$, as well as for the ratio of cross sections between 7, 8 and $14 \mathrm{TeV}$ and for the production of heavy top-like fermions. Previous phenomenological studies of the total $t \bar{t}$ cross sections, based on different approximations to the full NNLO calculation were presented in refs. [7-17].

As a first phenomenological application of the full NNLO calculation we study the impact of top quark cross section data in the parton distribution analysis. Indeed, top quark production is directly sensitive to the large- $x$ gluon PDF, which at present is affected by 


\begin{tabular}{|c|c|c|c|c|}
\hline & TeVatron & LHC 7 TeV & LHC 8 TeV & LHC $14 \mathrm{TeV}$ \\
\hline$g g$ & $15.4 \%$ & $84.8 \%$ & $86.2 \%$ & $90.2 \%$ \\
$q g+\bar{q} g$ & $-1.7 \%$ & $-1.6 \%$ & $-1.1 \%$ & $0.5 \%$ \\
$q q$ & $86.3 \%$ & $16.8 \%$ & $14.9 \%$ & $9.3 \%$ \\
\hline
\end{tabular}

Table 1. The relative contribution of the various partonic sub-channels to the NNLO+NNLL cross section for different colliders and collider energies, computed with the MSTW2008NNLO PDFs. We loosely label with $q q$ the sum of all processes without gluons in the initial state.

substantial uncertainties. In turn, large- $x$ gluons play an important role in theoretical predictions of many BSM scenarios like gluino pair production [18], high-mass Kaluza-Klein graviton production [19-21], resonances in the $t \bar{t}$ invariant mass spectrum [22, 23], quark compositeness in inclusive jet and dijet production [24-27] and many others. The availability of the full NNLO calculation makes top quark pair production the only hadron collider process that is both sensitive to the gluon and can be consistently included in a NNLO PDF fit without any approximations. Hadronic constraints on the gluon PDF are provided also by inclusive jet and dijet production [28-31] and isolated photon production $[32,33]$, though these two processes are only known to NLO and affected by substantial scale uncertainties. ${ }^{1}$

The focus of this paper is, on the one hand, to provide an up-to-date summary of the theoretical uncertainties on the total $t \bar{t}$ cross section, and on the other hand, to show how top quark data can be used to constrain the large- $x$ gluon PDF. Indeed, unlike the Tevatron, top quark pair production at the LHC is dominated by $g g$ scattering, thus providing a complementary probe of the gluon PDF. As shown in table 1, at the LHC the relative contribution of the $g g$ subprocess is between $85 \%$ and $90 \%$ depending on the beam energy, with $q q$ being about 10-15\%, almost the opposite of the Tevatron.

To illustrate the range of Bjorken- $x$ 's to which the top cross section is sensitive, the correlation [35] between the top quark production cross section and the gluon and the up quark PDFs is shown in figure 1 for the various cases that we will discuss in the paper: Tevatron Run II, LHC 7, 8 and $14 \mathrm{TeV}$. A correlation whose absolute magnitude is close to 1 indicates that variations of PDFs with a particular value of $x$ will in turn translate into cross-section variations. It is clear from figure 1 that for the LHC the top quark cross section directly probes the gluon in the range of $x$ between $x=0.1$ and $x=0.5$, where gluon PDF uncertainties are relatively large.

The outline of this paper is as follows. In section 2 we discuss the settings of the calculation and the treatment of the various theoretical uncertainties. In section 3 we provide up-to-date predictions for the $t \bar{t}$ cross section at the Tevatron and LHC and compare with the most recent experimental data. In section 4 we quantify the impact of the available top data on the gluon PDF, show how it reduces the gluon PDF's large- $x$ uncertainties, and study the phenomenological consequences of this improvement. In section 5 we provide up-to-date predictions for the ratio of top quark cross sections between 7,8 and $14 \mathrm{TeV}$ and in section 6 we provide predictions for a heavy top-like fermion $T$. 

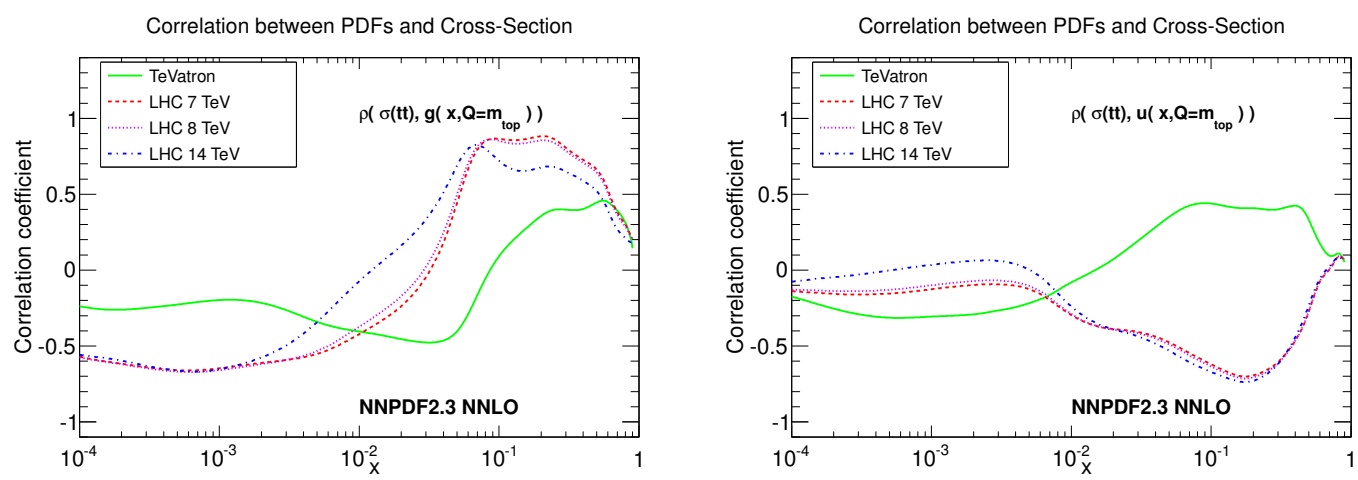

Figure 1. The correlation between the gluon PDF (left plot) and the up quark PDF (right plot) from NNPDF2.3 with the total NNLO+NNLL top quark production cross sections at the Tevatron and the LHC for different center of mass energies. The correlations are computed for $Q=m_{t}=173.3 \mathrm{GeV}$.

\section{Settings}

In the following we present the settings of the computation. We use the program Top++, v2.0 [36] to evaluate the total top quark production cross section at NNLO+NNLL accuracy. We use as input the most up-to-date NNLO PDF sets from each PDF collaboration: ABM11 [37], CT10 [38], HERAPDF1.5 [39], JR09 [40] MSTW08 [41] and NNPDF2.3 [42]. The value of $\alpha_{s}\left(M_{Z}\right)$ is set to the preferred value of each group, respectively $0.1134,0.118$, $0.1176,0.120,0.1171$ and 0.118 . For NNPDF2.3 we use the set with a maximum of $N_{f}=5$ active flavors. When providing the predictions for each PDF set, we will use the default $\alpha_{s}$ in each case, while later in section 3.3 we will explore the dependence of the results on the strong coupling as $\alpha_{s}$ is varied. A detailed benchmark comparison of these five NNLO PDF sets was recently presented in [43], where the similarities and differences between each of the five sets are discussed.

We consider the following sources of theoretical uncertainties in the top quark pair production cross section:

- Higher perturbative orders.

The central scales of the NNLO+NNLL computation are set to $\mu_{R}=\mu_{F}=m_{t}$. As customary, we explore the effect of missing higher perturbative orders by varying the scales independently by a factor of two upwards and downwards, with the constraint that the ratio of the two scales can never be larger than two. The envelope of the resulting cross section defines the scale uncertainty of the computation. As shown in ref. [1], scale variations at the LHC with the full NNLO+NNLL result are substantially smaller that with the NLO or previous approximated NNLO computations.

- Parton Distributions.

We use the corresponding prescription from each group to provide the $68 \%$ confidence level PDF uncertainties. For the Hessian sets, we use the asymmetric expression for PDF uncertainties [44]. The CT10 errors are rescaled by a factor 1.642 since 
they are provided as $90 \% \mathrm{CL}$. In the case of HERAPDF1.5, we consider only the experimental uncertainties, but not the model and parametrization uncertainties. ${ }^{2}$ Parton distributions are accessed through the LHAPDF interface, version 5.8.9 [48].

- Strong coupling constant.

We assume the $68 \%$ CL uncertainty on $\alpha_{s}\left(M_{Z}\right)$ to be $\Delta \alpha_{s}=0.0007$, as indicated by the 2012 PDG average [45]. For each PDF set, we add in quadrature PDF and $\alpha_{s}$ uncertainties, except for ABM11 and JR09, where $\alpha_{s}$ errors are already part of the total PDF uncertainty, and thus we avoid double counting. For Hessian PDF sets the addition in quadrature of PDF and $\alpha_{s}$ uncertainties is known to be exact [46], while for Monte Carlo sets it is a good approximation [47]. The slope of the cross section with $\alpha_{s}$ for each PDF set is extracted from a linear fit in a common range of $\alpha_{s}\left(M_{Z}\right)$ between 0.116 and 0.120 , close to the PDG average and where all PDF groups provide predictions.

- Top quark mass.

We take as central value $m_{t}=173.3 \mathrm{GeV}$, with an uncertainty of $\Delta m_{t}= \pm 1 \mathrm{GeV}$. This is consistent both with the latest Tevatron average, ${ }^{3} m_{t}=173.20 \pm 0.87 \mathrm{GeV}$, as well as with the 2012 PDG average [45] of the top quark mass $m_{t}=173.5 \pm$ $0.6 \pm 0.8 \mathrm{GeV}$. These masses are to be understood as pole masses. To determine the top-mass related uncertainty $\delta_{\mathrm{m}_{\mathrm{t}}}$ of the top cross section, we compute the central value of the cross-section for top masses in a $1 \mathrm{GeV}$ range around the central value $m_{t}=173.3$.

Following the recommendations of the Higgs Cross section Working Group [50] regarding theoretical uncertainties, we adopt the most conservative method to combine them: we add all parametric uncertainties (PDFs, $\alpha_{s}$ and $m_{t}$ ) in quadrature and then the total parametric error is added linearly to the scale uncertainty to define the total theoretical uncertainty.

\section{The top cross-section: measurements, predictions and uncertainties}

With the settings detailed in the previous section, we have computed the cross sections for the Tevatron and the LHC and their associated uncertainties. The most precise experimental measurements of the top cross section are collected in table 2, while the theoretical predictions are collected in tables 3 to 6 . In each case, we provide the NNLO+NNLL predictions for the total top quark cross section, for all the PDFs considered, and with the various sources of theoretical uncertainties. The default value of $\alpha_{s}\left(M_{Z}\right)$ from each PDF set has been used in the computation of the central predictions. Also included in these tables are the best available experimental measurements at the various colliders.

\footnotetext{
${ }^{2}$ If the HERAPDF1.5 uncertainties for the $t \bar{t}$ cross section include the model and parametrization uncertainties the total PDF uncertainty increases to about 20\% [43].

${ }^{3}$ See [49], and the update prepared for the 2013 Rencontres de Moriond QCD, presented by G. Petrillo.
} 


\begin{tabular}{|c|c|c|c|c|c|}
\hline Measurement & $\sigma_{t \bar{t}}(\mathrm{pb})$ & stat. (pb) & sys. (pb) & lumi. (pb) & total (pb) \\
\hline Tevatron CDF+D0 (Ref. [51]) & 7.65 & \pm 0.20 & \pm 0.29 & \pm 0.22 & $7.65 \pm 0.42(5.5 \%)$ \\
Atlas 7 TeV (Ref. [52]) & 177 & \pm 3 & ${ }_{-7}^{+8}$ & \pm 7 & $177_{-11(+6.2 \%)}^{+10}(+5.6 \%)$ \\
CMS 7 TeV (Ref. [53]) & 160.9 & \pm 2.5 & ${ }_{-5.0}^{+5.1}$ & \pm 3.6 & $160.9 \pm 6.6(4.0 \%)$ \\
Atlas 8 TeV (Ref. [54]) & 241 & \pm 2 & \pm 31 & \pm 9 & $241 \pm 32(13.0 \%)$ \\
CMS 8 TeV (Refs. [55, 56]) & 227 & \pm 3 & \pm 11 & \pm 10 & $227 \pm 15(6.7 \%)$ \\
\hline
\end{tabular}

Table 2. The experimental measurements from the Tevatron and LHC 7 and $8 \mathrm{TeV}$ used in this paper. The statistical, systematic and luminosity uncertainties are added in quadrature for the total uncertainty in the last column. See the text for more details.

For the Tevatron, we have used the most up-to-date CDF and D0 combinations [51] with $8.8 \mathrm{fb}^{-1}$ of data, see table 2. For $\mathrm{LHC} 7 \mathrm{TeV}$, we quote separately the two most precise measurements from ATLAS and CMS. From ATLAS we use the combination of all channels with luminosities between 0.70 and $1.02 \mathrm{fb}^{-1}$ [52], see table 2, while for CMS we use the single most precise measurement, ${ }^{4}$ obtained in the dilepton channel with a luminosity of $2.03 \mathrm{fb}^{-1}$ [53], also given in table 2 . Note also that a preliminary combination of all channels between ATLAS and CMS for an integrated luminosity of $1.0 \mathrm{fb}^{-1}$ has been presented in [57], yielding $\sigma(t \bar{t})=173.3 \pm 10.1 \mathrm{pb}$. For LHC $8 \mathrm{TeV}$, there are three available measurements, two from CMS, in the dilepton channel, with $2.4 \mathrm{fb}^{-1}$ [55] and in the lepton+jets channel with $2.8 \mathrm{fb}^{-1}$ [56], and one from ATLAS in the lepton+jets channel with, $5.8 \mathrm{fb}^{-1}$ [54]. We use the CMS dilepton and the ATLAS lepton+jets results, collected in table 2 .

In figure 2 we show the predictions from each PDF set compared to experimental data. The $x$-axis is the reference value of $\alpha_{s}$ for each PDF set. The inner error bars correspond to the linear sum of scale and PDF uncertainties, while the outer error bar is the total theoretical uncertainty including also the contributions from $\delta m_{t}$ and $\delta \alpha_{s}{ }^{5}$ Similar comparisons of the PDF and $\alpha_{s}$ sensitivity of $\sigma(t \bar{t})$, based on previous approximate NNLO calculations, have been reported in refs. [10-12].

In the remainder of this section we comment on various features of the above results.

\subsection{Comparison between the various PDF sets}

We notice that the results for NNPDF2.3, CT10, MSTW08 and HERAPDF1.5 are all close to each other, both in central values and theoretical uncertainties. JR09 leads to similar central values, despite the smaller value of $\alpha_{s}$, but is affected by larger $\mathrm{PDF}+\alpha_{s}$ uncertainties. On the other hand, the ABM11 predictions are lower compared to other sets and to experimental data. Figure 1 suggests that similarities or differences in the total

\footnotetext{
${ }^{4}$ In this case, given that the dependence of the measured total cross section with $m_{t}$ (which enters through the acceptance correction) is provided, we have rescaled the central value of the data to the same value of the top quark mass used for the theory computations, $m_{t}=173.3 \mathrm{GeV}$. Other cross-section measurements do not provide this information but rather include the $\delta_{m_{t}}$ uncertainty in the computation of the acceptances as part of the total systematic uncertainty.

${ }^{5}$ The inner error bar can thus be compared directly to previous phenomenological studies, which consider only PDF and scale uncertainties.
} 


\begin{tabular}{|c|c|c|c|c|c|c|}
\hline \multicolumn{7}{|c|}{ Tevatron Run II } \\
\hline PDF set & $\sigma_{t t}(\mathrm{pb})$ & $\delta_{\text {scale }}(\mathrm{pb})$ & $\delta_{\mathrm{PDF}}(\mathrm{pb})$ & $\delta_{\alpha_{s}}(\mathrm{pb})$ & $\delta_{\mathrm{m}_{\mathrm{t}}}(\mathrm{pb})$ & $\delta_{\text {tot }}(\mathrm{pb})$ \\
\hline ABM11 & 6.869 & $\begin{array}{ll}+0.104 & (+1.5 \%) \\
-0.174 & (-2.5 \%)\end{array}$ & $\begin{array}{ll}+0.157 & (+2.3 \%) \\
-0.157 & (-2.3 \%)\end{array}$ & $\begin{array}{ll}+0.000 & (+0.0 \%) \\
-0.000 & (-0.0 \%)\end{array}$ & $\begin{array}{ll}+0.207 & (+3.0 \%) \\
-0.201 & (-2.9 \%)\end{array}$ & $\begin{array}{ll}+0.364 & (+5.3 \%) \\
-0.429 & (-6.2 \%)\end{array}$ \\
\hline CT10 & 7.395 & $\begin{array}{ll}+0.116 & (+1.6 \%) \\
-0.210 & (-2.8 \%)\end{array}$ & $\begin{array}{ll}+0.270 & (+3.6 \%) \\
-0.203 & (-2.7 \%)\end{array}$ & $\begin{array}{ll}+0.136 & (+1.8 \%) \\
-0.136 & (-1.8 \%)\end{array}$ & $\begin{array}{ll}+0.235 & (+3.2 \%) \\
-0.227 & (-3.1 \%)\end{array}$ & $\begin{array}{ll}+0.499 & (+6.7 \%) \\
-0.544 & (-7.4 \%)\end{array}$ \\
\hline HERA1.5 & 7.624 & $\begin{array}{ll}+0.116 & (+1.5 \%) \\
-0.074 & (-1.0 \%)\end{array}$ & $\begin{array}{ll}+0.134 & (+1.8 \%) \\
-0.154 & (-2.0 \%)\end{array}$ & $\begin{array}{ll}+0.098 & (+1.3 \%) \\
-0.098 & (-1.3 \%)\end{array}$ & $\begin{array}{ll}+0.241 & (+3.2 \%) \\
-0.233 & (-3.1 \%)\end{array}$ & $\begin{array}{ll}+0.409 & (+5.4 \%) \\
-0.370 & (-4.9 \%)\end{array}$ \\
\hline JR09 & 7.174 & $\begin{array}{ll}+0.099 & (+1.4 \%) \\
-0.054 & (-0.8 \%)\end{array}$ & $\begin{array}{ll}+0.326 & (+4.6 \%) \\
-0.326 & (-4.6 \%)\end{array}$ & $\begin{array}{ll}+0.000 & (+0.0 \%) \\
-0.000 & (-0.0 \%)\end{array}$ & $\begin{array}{ll}+0.215 & (+3.0 \%) \\
-0.211 & (-2.9 \%)\end{array}$ & $\begin{array}{ll}+0.490 & (+6.8 \%) \\
-0.443 & (-6.2 \%)\end{array}$ \\
\hline MSTW08 & 7.164 & $\begin{array}{ll}+0.110 & (+1.5 \%) \\
-0.200 & (-2.8 \%)\end{array}$ & $\begin{array}{ll}+0.169 & (+2.4 \%) \\
-0.122 & (-1.7 \%)\end{array}$ & $\begin{array}{ll}+0.088 & (+1.2 \%) \\
-0.088 & (-1.2 \%)\end{array}$ & $\begin{array}{ll}+0.228 & (+3.2 \%) \\
-0.220 & (-3.1 \%)\end{array}$ & $\begin{array}{ll}+0.391 & (+5.5 \%) \\
-0.475 & (-6.6 \%)\end{array}$ \\
\hline NNPDF 2.3 & 7.258 & $\begin{array}{ll}+0.117 & (+1.6 \%) \\
-0.202 & (-2.8 \%)\end{array}$ & $\begin{array}{ll}+0.121 & (+1.7 \%) \\
-0.121 & (-1.7 \%)\end{array}$ & $\begin{array}{ll}+0.090 & (+1.2 \%) \\
-0.090 & (-1.2 \%)\end{array}$ & $\begin{array}{ll}+0.229 & (+3.1 \%) \\
-0.221 & (-3.0 \%)\end{array}$ & $\begin{array}{ll}+0.390 & (+5.4 \%) \\
-0.469 & (-6.5 \%)\end{array}$ \\
\hline $\mathrm{CDF}+\mathrm{D} 0$ & 7.65 & & & & & $\pm 0.42(5.5 \%)$ \\
\hline
\end{tabular}

Table 3. The NNLO+NNLL predictions for the total top quark pair cross-section at the Tevatron Run II, for all the PDFs considered, and with the various sources of theoretical uncertainties. The default value of $\alpha_{s}\left(M_{Z}\right)$ from each collaboration has been used in the computation of the central predictions. The four theoretical uncertainties are combined into a total theory error as discussed in the text. The lower row shows the best available experimental measurement.

cross section can be understood in terms of the large- $x$ behavior of the gluon PDF, together with the value of $\alpha_{s}$ used by each PDF set.

The agreement between CT, HERAPDF, MSTW and NNPDF can be traced back to (a) a similar default value of $\alpha_{s}$ used and (b) a similar large- $x$ gluon PDF [43]. On the other hand, regarding the differences between ABM11 and the other sets, we note (a) the smaller value of $\alpha_{s}$ used by ABM11 and (b) the softer large- $x$ gluon PDF in the region relevant for top quark production [43]. As shown below in figure 3, using a value for $\alpha_{s}$ closer to the PDG average would improve the agreement of the ABM11 set both with other PDF sets and with experimental data, though the ABM11 predictions at the LHC are still lower than that of other sets even for a common $\alpha_{s}$ value.

To understand the differences in the large- $x$ gluon in ABM11 as compared to other sets, refs. [61, 62] have suggested that the reason is that, keeping everything else fixed, the use of a fixed-flavor number scheme in a fit to deep-inelastic data (such as ABM11) leads to a softer large- $x$ gluon as compared to the gluon obtained in PDF fits based on variable-flavor number schemes [58-60]. On the other hand, the differences between the various implementations of variable-flavor number schemes translate into much reduced differences in the PDFs [61] and thus into the top cross sections.

In order to quantify the agreement of data and theory, in the absence of theoretical uncertainties, we compute the $\chi^{2}$ for the central values of the five PDF sets under consideration and for the five available data points of table 2: one for the Tevatron, two for LHC $7 \mathrm{TeV}$ and two for LHC $8 \mathrm{TeV}$. For each of the five PDF sets studied, the $\chi^{2}$ is defined as

$$
\chi^{2}=\sum_{i=1}^{N_{\text {dat }}} \frac{\left(\sigma_{t \bar{t}}^{(\exp )}-\sigma_{t \bar{t}}^{(\mathrm{th})}\right)^{2}}{\delta_{\mathrm{tot}}^{(\exp ) 2}},
$$

with $N_{\text {dat }}=5$. We consider all five data points as fully uncorrelated. ${ }^{6}$ In addition,

\footnotetext{
${ }^{6}$ This is a necessary approximation, but as discussed in [57] there is some degree of correlation between
} 


\begin{tabular}{|c|c|c|c|c|c|c|}
\hline \multicolumn{7}{|c|}{$\mathrm{LHC} 7 \mathrm{TeV}$} \\
\hline PDF set & $\sigma_{t t}(\mathrm{pb})$ & $\delta_{\text {scale }}(\mathrm{pb})$ & $\delta_{\mathrm{PDF}}(\mathrm{pb})$ & $\delta_{\alpha_{s}}(\mathrm{pb})$ & $\delta_{\mathrm{m}_{\mathrm{t}}}(\mathrm{pb})$ & $\delta_{\text {tot }}(\mathrm{pb})$ \\
\hline ABM11 & 135.8 & $\begin{array}{ll}+3.5 & (+2.6 \%) \\
-4.2 & (-3.1 \%)\end{array}$ & $\begin{array}{ll}+6.4 & (+4.7 \%) \\
-6.4(-4.7 \%)\end{array}$ & $\begin{array}{ll}+0.0 & (+0.0 \%) \\
-0.0 & (-0.0 \%)\end{array}$ & $\begin{array}{ll}+4.3(+3.2 \%) \\
-4.2(-3.1 \%)\end{array}$ & $\begin{array}{ll}+11.2 & (+8.2 \%) \\
-11.8 & (-8.7 \%)\end{array}$ \\
\hline CT10 & 172.5 & $\begin{array}{ll}+4.6 & (+2.7 \%) \\
-6.0 & (-3.5 \%)\end{array}$ & $\begin{array}{ll}+8.0 & (+4.6 \%) \\
-6.5 & (-3.8 \%)\end{array}$ & $\begin{array}{ll}+3.7 & (+2.2 \%) \\
-3.7 & (-2.2 \%)\end{array}$ & $\begin{array}{ll}+5.3 & (+3.1 \%) \\
-5.1 & (-3.0 \%)\end{array}$ & $\begin{array}{ll}+14.9 & (+8.6 \%) \\
-15.0 & (-8.7 \%)\end{array}$ \\
\hline HERA1.5 & 177.2 & $\begin{array}{ll}+4.8 & (+2.7 \%) \\
-4.2 & (-2.3 \%)\end{array}$ & $\begin{array}{ll}+4.0 & (+2.3 \%) \\
-6.4 & (-3.6 \%)\end{array}$ & $\begin{array}{ll}+3.0 & (+1.7 \%) \\
-3.0 & (-1.7 \%)\end{array}$ & $\begin{array}{ll}+5.4 & (+3.1 \%) \\
-5.2( & (-2.9 \%)\end{array}$ & $\begin{array}{ll}+12.2 & (+6.9 \%) \\
-12.9 & (-7.3 \%)\end{array}$ \\
\hline JR09 & 167.0 & $\begin{array}{ll}+3.9 & (+2.4 \%) \\
-3.3 & (-2.0 \%)\end{array}$ & $\begin{array}{ll}+12.6 & (+7.6 \%) \\
-12.6 & (-7.6 \%)\end{array}$ & $\begin{array}{ll}+0.0 & (+0.0 \%) \\
-0.0 & (-0.0 \%)\end{array}$ & $\begin{array}{ll}+4.5 & (+2.7 \%) \\
-4.3(-2.6 \%)\end{array}$ & $\begin{array}{ll}+17.3 & (+10.4 \%) \\
-16.6 & (-9.9 \%)\end{array}$ \\
\hline MSTW08 & 172.0 & $\begin{array}{ll}+4.4 & (+2.6 \%) \\
-5.8 & (-3.4 \%)\end{array}$ & $\begin{array}{ll}+4.7 & (+2.7 \%) \\
-4.7 & (-2.7 \%)\end{array}$ & $\begin{array}{ll}+2.9 & (+1.7 \%) \\
-2.9 & (-1.7 \%)\end{array}$ & $\begin{array}{ll}+5.3 & (+3.1 \%) \\
-5.1 & (-3.0 \%)\end{array}$ & $\begin{array}{ll}+12.1 & (+7.0 \%) \\
-13.4 & (-7.8 \%)\end{array}$ \\
\hline NNPDF 2.3 & 172.7 & $\begin{array}{ll}+4.6 & (+2.7 \%) \\
-6.0 & (-3.5 \%)\end{array}$ & $\begin{array}{ll}+5.2 & (+3.0 \%) \\
-5.2 & (-3.0 \%)\end{array}$ & $\begin{array}{ll}+2.7 & (+1.6 \%) \\
-2.7 & (-1.6 \%)\end{array}$ & $\begin{array}{ll}+5.3 & (+3.1 \%) \\
-5.2 & (-3.0 \%)\end{array}$ & $\begin{array}{ll}+12.5 & (+7.2 \%) \\
-13.7 & (-8.0 \%)\end{array}$ \\
\hline $\begin{array}{c}\text { ATLAS } \\
\text { CMS }\end{array}$ & $\begin{array}{c}177 \\
160.9\end{array}$ & & & & & $\begin{array}{r}+10(+5.6 \%) \\
-11(-6.2 \%) \\
\pm 6.6(4.0 \%)\end{array}$ \\
\hline
\end{tabular}

Table 4. Same as table 3 for LHC $7 \mathrm{TeV}$.

\begin{tabular}{|c|c|c|c|c|c|c|}
\hline \multicolumn{7}{|c|}{ LHC $8 \mathrm{TeV}$} \\
\hline PDF set & $\sigma_{t t}(\mathrm{pb})$ & $\delta_{\text {scale }}(\mathrm{pb})$ & $\delta_{\mathrm{PDF}}(\mathrm{pb})$ & $\delta_{\alpha_{s}}(\mathrm{pb})$ & $\delta_{\mathrm{m}_{\mathrm{t}}}(\mathrm{pb})$ & $\delta_{\text {tot }}(\mathrm{pb})$ \\
\hline ABM11 & 198.6 & $\begin{array}{ll}+5.0 & (+2.5 \%) \\
-6.2 & (-3.1 \%)\end{array}$ & $\begin{array}{ll}+8.5 & (+4.3 \%) \\
-8.5 & (-4.3 \%)\end{array}$ & $\begin{array}{ll}+0.0 & (+0.0 \%) \\
-0.0 & (-0.0 \%)\end{array}$ & $\begin{array}{ll}+6.1 & (+3.1 \%) \\
-5.9 & (-3.0 \%)\end{array}$ & $\begin{array}{ll}+15.5 & (+7.8 \%) \\
-16.6 & (-8.4 \%)\end{array}$ \\
\hline CT10 & 246.3 & $\begin{array}{ll}+6.4 & (+2.6 \%) \\
-8.6(-3.5 \%)\end{array}$ & $\begin{array}{ll}+10.1 & (+4.1 \%) \\
-8.2 & (-3.3 \%)\end{array}$ & $\begin{array}{ll}+4.9 & (+2.0 \%) \\
-4.9(-2.0 \%)\end{array}$ & $\begin{array}{ll}+7.4 & (+3.0 \%) \\
-7.1 & (-2.9 \%)\end{array}$ & $\begin{array}{ll}+19.8 & (+8.1 \%) \\
-20.5 & (-8.3 \%)\end{array}$ \\
\hline HERA1.5 & 252.7 & $\begin{array}{ll}+6.5 & (+2.6 \%) \\
-5.9 & (-2.3 \%)\end{array}$ & $\begin{array}{ll}+5.4 & (+2.1 \%) \\
-8.6 & (-3.4 \%)\end{array}$ & $\begin{array}{ll}+4.0 & (+1.6 \%) \\
-4.0 & (-1.6 \%)\end{array}$ & $\begin{array}{ll}+7.5 & (+3.0 \%) \\
-7.3 & (-2.9 \%)\end{array}$ & $\begin{array}{l}+16.6(+6.6 \%) \\
-17.8(-7.1 \%)\end{array}$ \\
\hline JR09 & 238.0 & $\begin{array}{ll}+2.1 & (+0.9 \%) \\
-4.6 & (-1.9 \%)\end{array}$ & $\begin{array}{l}+15.8(+6.6 \%) \\
-15.8(-6.6 \%)\end{array}$ & $\begin{array}{ll}+0.0 & (+0.0 \%) \\
-0.0 & (-0.0 \%)\end{array}$ & $\begin{array}{l}+6.3(+2.7 \%) \\
-6.2(-2.6 \%)\end{array}$ & $\begin{array}{l}+19.2(+8.1 \%) \\
-21.6(-9.1 \%)\end{array}$ \\
\hline MSTW08 & 245.8 & $\begin{array}{ll}+6.2 & (+2.5 \%) \\
-8.4(-3.4 \%)\end{array}$ & $\begin{array}{ll}+6.2 & (+2.5 \%) \\
-6.2 & (-2.5 \%)\end{array}$ & $\begin{array}{ll}+4.0 & (+1.6 \%) \\
-4.0 & (-1.6 \%)\end{array}$ & $\begin{array}{ll}+7.4 & (+3.0 \%) \\
-7.1 & (-2.9 \%)\end{array}$ & $\begin{array}{l}+16.6(+6.8 \%) \\
-18.7(-7.6 \%)\end{array}$ \\
\hline NNPDF2.3 & 248.1 & $\begin{array}{ll}+6.4 & (+2.6 \%) \\
-8.7 & (-3.5 \%) \\
\end{array}$ & $\begin{array}{ll}+6.6 & (+2.7 \%) \\
-6.6(-2.7 \%) \\
\end{array}$ & $\begin{array}{ll}+3.7 & (+1.5 \%) \\
-3.7 & (-1.5 \%) \\
\end{array}$ & $\begin{array}{ll}+7.5 & (+3.0 \%) \\
-7.2(-2.9 \%)\end{array}$ & $\begin{array}{ll}+17.1 & (+6.9 \%) \\
-19.1 & (-7.7 \%)\end{array}$ \\
\hline ATLAS & 241.0 & & & & & $\pm 32.0(13.3 \%)$ \\
\hline CMS & 227.0 & & & & & $\pm 15.0(6.6 \%)$ \\
\hline
\end{tabular}

Table 5. Same as table 3 for LHC $8 \mathrm{TeV}$.

\begin{tabular}{|c|c|c|c|c|c|c|}
\hline \multicolumn{7}{|c|}{$\mathrm{LHC} 14 \mathrm{TeV}$} \\
\hline PDF set & $\sigma_{t t}(\mathrm{pb})$ & $\delta_{\text {scale }}(\mathrm{pb})$ & $\delta_{\mathrm{PDF}}(\mathrm{pb})$ & $\delta_{\alpha_{s}}(\mathrm{pb})$ & $\delta_{\mathrm{m}_{\mathrm{t}}}(\mathrm{pb})$ & $\delta_{\text {tot }}(\mathrm{pb})$ \\
\hline ABM11 & 832.0 & $\begin{array}{ll}+18.7 & (+2.2 \%) \\
-27.4 & (-3.3 \%)\end{array}$ & $\begin{array}{ll}+25.1 & (+3.0 \%) \\
-25.1 & (-3.0 \%)\end{array}$ & $\begin{array}{ll}+0.0 & (+0.0 \%) \\
-0.0 & (-0.0 \%)\end{array}$ & $\begin{array}{ll}+23.3 & (+2.8 \%) \\
-22.5 & (-2.7 \%)\end{array}$ & $\begin{array}{ll}+52.9 & (+6.4 \%) \\
-61.1 & (-7.3 \%)\end{array}$ \\
\hline CT10 & 952.8 & $\begin{array}{ll}+23.3 & (+2.4 \%) \\
-34.5 & (-3.6 \%)\end{array}$ & $\begin{array}{ll}+22.4 & (+2.3 \%) \\
-19.9 & (-2.1 \%)\end{array}$ & $\begin{array}{ll}+14.0 & (+1.5 \%) \\
-14.0 & (-1.5 \%)\end{array}$ & $\begin{array}{ll}+26.1 & (+2.7 \%) \\
-25.2 & (-2.6 \%)\end{array}$ & $\begin{array}{ll}+60.3 & (+6.3 \%) \\
-69.5 & (-7.3 \%)\end{array}$ \\
\hline HERA1.5 & 970.5 & $\begin{array}{ll}+22.1 & (+2.3 \%) \\
-22.0 & (-2.3 \%)\end{array}$ & $\begin{array}{ll}+15.3 & (+1.6 \%) \\
-25.7 & (-2.6 \%)\end{array}$ & $\begin{array}{ll}+12.8 & (+1.3 \%) \\
-12.8 & (-1.3 \%)\end{array}$ & $\begin{array}{ll}+26.4 & (+2.7 \%) \\
-25.6 & (-2.6 \%)\end{array}$ & $\begin{array}{ll}+55.2 & (+5.7 \%) \\
-60.5 & (-6.2 \%)\end{array}$ \\
\hline JR09 & 906.5 & $\begin{array}{ll}+16.7 & (+1.8 \%) \\
-17.0 & (-1.9 \%)\end{array}$ & $\begin{array}{ll}+35.5 & (+3.9 \%) \\
-35.5 & (-3.9 \%)\end{array}$ & $\begin{array}{ll}+0.0 & (+0.0 \%) \\
-0.0 & (-0.0 \%)\end{array}$ & $\begin{array}{ll}+24.7 & (+2.7 \%) \\
-23.9 & (-2.6 \%)\end{array}$ & $\begin{array}{ll}+60.0 & (+6.6 \%) \\
-59.8 & (-6.6 \%)\end{array}$ \\
\hline MSTW08 & 953.6 & $\begin{array}{ll}+22.7 & (+2.4 \%) \\
-33.9 & (-3.6 \%)\end{array}$ & $\begin{array}{ll}+16.2 & (+1.7 \%) \\
-17.8 & (-1.9 \%)\end{array}$ & $\begin{array}{ll}+12.8 & (+1.3 \%) \\
-12.8 & (-1.3 \%)\end{array}$ & $\begin{array}{ll}+26.1 & (+2.7 \%) \\
-25.3 & (-2.7 \%)\end{array}$ & $\begin{array}{ll}+56.3 & (+5.9 \%) \\
-66.8 & (-7.0 \%)\end{array}$ \\
\hline NNPDF 2.3 & 977.5 & $\begin{array}{ll}+23.6 & (+2.4 \%) \\
-35.4 & (-3.6 \%)\end{array}$ & $\begin{array}{ll}+16.4 & (+1.7 \%) \\
-16.4 & (-1.7 \%)\end{array}$ & $\begin{array}{ll}+12.2 & (+1.3 \%) \\
-12.2 & (-1.3 \%)\end{array}$ & $\begin{array}{ll}+26.9 & (+2.8 \%) \\
-26.1 & (-2.7 \%)\end{array}$ & $\begin{array}{ll}+57.4 & (+5.9 \%) \\
-68.5 & (-7.0 \%)\end{array}$ \\
\hline
\end{tabular}

Table 6. Same as table 3 for LHC $14 \mathrm{TeV}$.

to quantify the overall consistency of theoretical predictions with experimental data, we define a pull estimator as follows,

$$
P=\frac{1}{N_{\text {dat }}} \sum_{i=1}^{N_{\text {dat }}} \frac{\left(\sigma_{t \bar{t}}^{(\exp )}-\sigma_{t \bar{t}}^{(\mathrm{th})}\right)^{2}}{\delta_{\text {tot }}^{(\exp ) 2}+\delta_{\text {tot }}^{(\mathrm{th}) 2}},
$$

the ATLAS and CMS data, as there is between LHC data at different c.m. energies. 

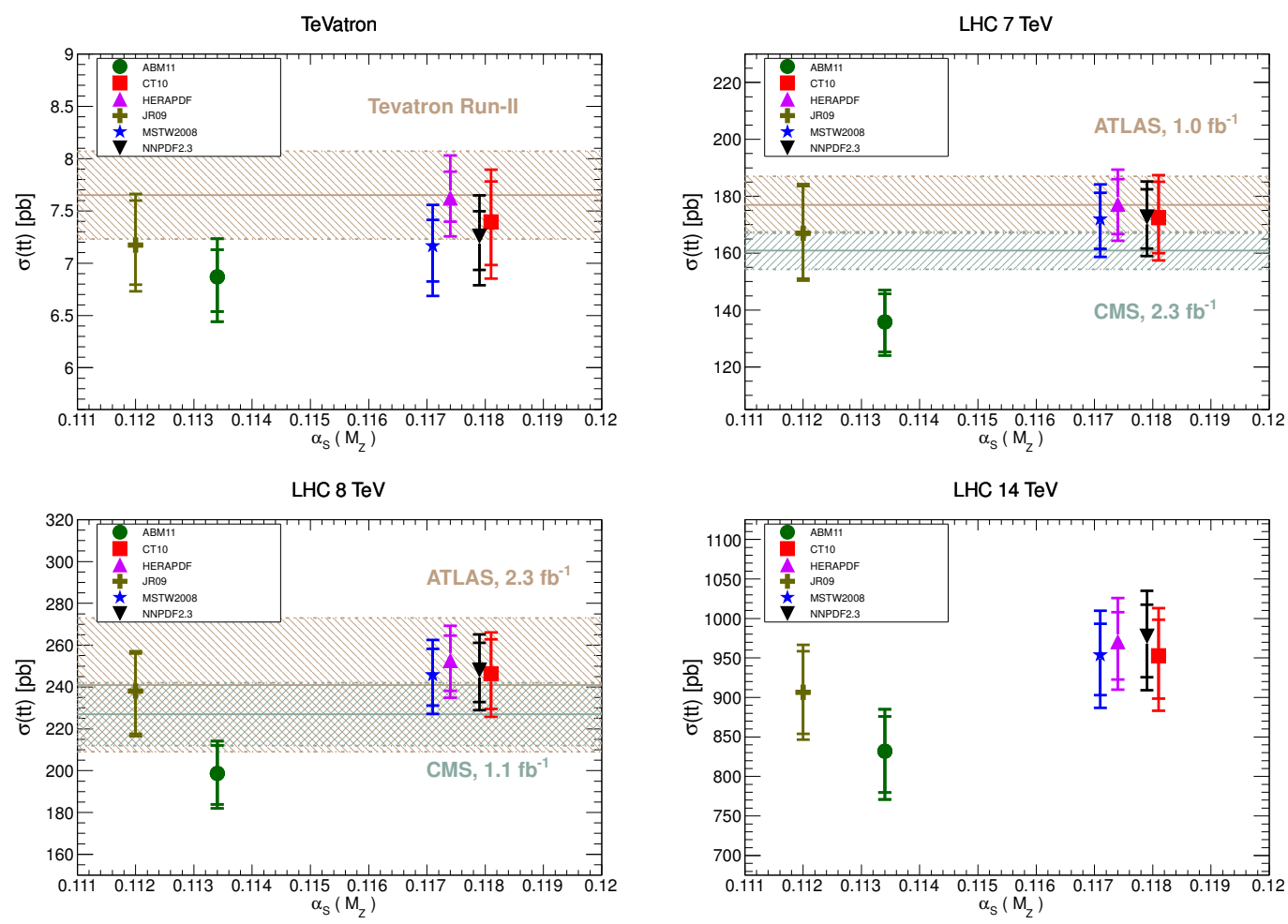

Figure 2. The best predictions from each PDF set compared to experimental data, as a function of the default $\alpha_{s}\left(M_{Z}\right)$ value. The inner error bar corresponds to the linear sum of PDF and scale uncertainties, while the outer error bar is the total theoretical uncertainty, computed as described in the text.

where now in the denominator we add in quadrature the experimental and theoretical total uncertainties. For simplicity we have symmetrized the total theoretical systematic error for each PDF set.

The results for both the $\chi^{2}$ and the pull are summarized in table 7 . We provide both the total $\chi^{2}$ and the individual contribution from the data at the Tevatron, LHC 7 and $8 \mathrm{TeV}$. As we can see, most PDF sets provide a good description of the total top quark cross section data, with $\chi^{2} / N_{\text {dat }} \sim 1$. The pull also shows the good consistency between experimental data and theory prediction for most of the PDF sets considered.

\subsection{Uncertainty due to the value of $m_{t}$}

The uncertainty on the value of the top quark mass is now a substantial fraction of the overall systematic uncertainty in the total $t \bar{t}$ cross section. In table 8 we compare, for the NNPDF2.3 set, the total theory uncertainty with and without including the uncertainty due to the top quark mass. We see that at the LHC, an uncertainty of $\delta m_{t}= \pm 1 \mathrm{GeV}$ translates into an increase between 1 and $1.5 \%$ of the total theory uncertainty, and into a somewhat larger increase at the Tevatron. Given that PDFs, scale and $m_{t}$ uncertainties are all now of similar size, the total theory error would be only slightly reduced if one assumed that the uncertainty due to $m_{t}$ could be completely neglected, for example after more precise measurements of this parameter at the LHC. 


\begin{tabular}{|c|c|c|c|c|c|c|}
\hline & $\chi_{\text {tev }}^{2}$ & $\chi_{\text {lhc7 }}^{2}$ & $\chi_{\text {lhc8 }}^{2}$ & $\chi_{\text {tot }}^{2}$ & $\chi_{\text {tot }}^{2} / N_{\text {dat }}$ & $\mathrm{P}$ \\
\hline AMB11 & 3.5 & 31.4 & 5.3 & 40.2 & 8.0 & 3.2 \\
CT10 & 0.4 & 3.3 & 1.7 & 5.3 & 1.1 & 0.3 \\
HERAPDF15 & 0.0 & 6.1 & 3.1 & 9.2 & 1.8 & 0.5 \\
MSTW08 & 1.3 & 3.1 & 1.6 & 6.0 & 1.2 & 0.4 \\
NNPDF2.3 & 0.9 & 3.4 & 2.0 & 6.3 & 1.3 & 0.4 \\
\hline
\end{tabular}

Table 7. The $\chi^{2}$ between data and theory, eq. (3.1), for all the five PDF sets, both for the total dataset and split into colliders. Let us recall that the standard deviation of the $\chi^{2}$ distribution for $N_{\text {dat }}=5$ data points is $\sqrt{2 N_{\text {dat }}}=3.1$. The last column show the pull eq. (3.2) between experimental data and theory predictions.

\begin{tabular}{|c|c|c|c|}
\hline Collider & $\sigma_{t t}(\mathrm{pb})$ & $\delta_{\mathrm{PDF}+\text { scales }+\alpha_{\mathrm{s}}}(\mathrm{pb})$ & $\delta_{\text {tot }}(\mathrm{pb})$ \\
\hline Tevatron & 7.258 & $\begin{array}{ll}+0.267 & (+3.7 \%) \\
-0.352 & (-4.9 \%)\end{array}$ & ${ }_{-0.469}^{+0.390}(+5.4 \%)$ \\
\hline LHC $7 \mathrm{TeV}$ & 172.7 & $\begin{array}{l}+10.4(+6.0 \%) \\
-11.8(-6.8 \%)\end{array}$ & $\begin{array}{ll}+12.5 & (+7.2 \%) \\
-13.7 & (-8.0 \%)\end{array}$ \\
\hline LHC $8 \mathrm{TeV}$ & 248.1 & $\begin{array}{l}+14.0(+5.6 \%) \\
{ }_{-16.2}(-6.5 \%)\end{array}$ & ${ }_{-191}^{+17.1}(+6.9 \%)$ \\
\hline LHC $14 \mathrm{TeV}$ & 977.5 & $\begin{array}{l}+44.1(+4.5 \%) \\
{ }_{-55.8}(-5.7 \%)\end{array}$ & $\begin{array}{ll}+57.4 & (+5.9 \%) \\
-68.5 & (-7.0 \%)\end{array}$ \\
\hline
\end{tabular}

Table 8. The NNLO+NNLL predictions for the total top quark pair cross-section at the Tevatron and the LHC, with NNPDF2.3 as input. The third column shows the theoretical uncertainty when $\delta m_{t}$ is not taken into account, while the last column is the total theoretical uncertainty (the same as in tables 3 to 6$)$. At the LHC, an uncertainty of $\delta m_{t}= \pm 1 \mathrm{GeV}$ translates into an increase between 1 and $1.5 \%$ of the total theory uncertainty, and a bit more at the Tevatron.

\subsection{Uncertainty due to the value of $\alpha_{s}$}

In figure 3 we show the dependence with $\alpha_{s}\left(M_{Z}\right)$ of the NNLO+NNLL cross sections for each of the various PDF sets. ${ }^{7}$ We use consistently the same value of $\alpha_{s}\left(M_{Z}\right)$ in the partonic cross sections and in the PDFs, using all the range of varying $\alpha_{s}$ PDFs provided by each group. We also include for reference the best experimental data. The slope with $\alpha_{s}$ is similar for each of the PDF sets. One can see that a variation of $\Delta \alpha_{s}$ by 0.001 increases the cross section by about $0.13 \mathrm{pb}(\sim 1.8 \%)$ at the Tevatron, $4 \mathrm{pb}(\sim 2.3 \%)$ at LHC $7 \mathrm{TeV}, 6 \mathrm{pb}(\sim 2.4 \%)$ at $8 \mathrm{TeV}$ and $20 \mathrm{pb}(\sim 2.0 \%)$ at $14 \mathrm{TeV}$, with small differences between PDF sets.

It is also worth noticing that the $\alpha_{s}$ dependence of the total cross section reported in our tables is slightly larger than what one would naively estimate based on the power counting of the partonic cross section. The reason is that, in the range of $x$ relevant for top quark production, the value of the gluon density is larger for PDF fits with a larger $\alpha_{s}$. This is shown in figure 4, for the specific case of NNPDF2.3, but the behavior is similar for other sets: larger $\alpha_{s}$ leads to smaller $g(x)$ in the small/medium- $x$ region that is controlled by deep-inelastic HERA data, while DGLAP evolution and the momentum

\footnotetext{
${ }^{7}$ Except for the case of JR09, where no varying- $\alpha_{s}$ PDF sets are provided.
} 
sum rule balance this reduction with an increase at larger $x$. In figure 4 we also show the corresponding plot for the case of the up quark density, relevant at the Tevatron. In this case the correlation with $\alpha_{s}$ is rather less marked and of opposite trend as in the case of the gluon.

The net effect of the positive correlation between the gluon PDF and $\alpha_{s}$ at large- $x$ is an enhanced sensitivity to $\alpha_{s}$ of the $t \bar{t}$ cross section, about $20 \%$ larger than what one would have obtained by keeping the PDFs fixed while changing only $\alpha_{s}$ in the partonic matrix element. ${ }^{8}$ This fact, together with the small, $2-3 \%$, scale uncertainty of the full NNLO+NNLL result, suggest that the top production cross section could provide a useful independent determination of $\alpha_{s}\left(M_{Z}\right)$. Such extraction would be analogous to the determinations of $m_{t}$ from the same total cross section [15-17, 63-65]. The strong coupling can also be determined at the Tevatron and the LHC from jet production [66-68], but results are affected by sizable scale uncertainties from the lack of a complete NNLO calculation as well as by non-perturbative corrections. A first determination of $\alpha_{s}$ from the $t \bar{t}$ cross section, using previous approximate NNLO predictions, has been performed by the CMS Collaboration [69].

\section{Top quark data constraints on the gluon PDF}

The gluon PDF is one of the worse known partonic distributions. Deep-inelastic data constrain the gluon only indirectly, via scaling violations, and direct information comes only from collider inclusive jet production data [28-31]. Recently, the use of isolated photon data has also been advocated to pin down the gluon [32]. However, direct constrains at hadron collider from photon and jet data are affected by substantial scale uncertainties due to the missing full NNLO result, and are complicated by various non-perturbative uncertainties. The availability of a full NNLO calculation makes the total top pair cross section the only collider observable which, at present, is both directly sensitive to the gluon PDF and can be consistently included in a NNLO QCD analysis. The fact that non-perturbative corrections are much reduced in the total top quark production cross-section as compared to photons and jets is another good motivation to use tops as probes of the gluon PDF. The possibility of using top cross section data in PDF analysis was already discussed at the qualitative level in ref. [70]. More recently, ref. [16] provided a first estimate of the impact of top quark data on the gluon PDF based on approximate NNLO results.

In section 3 we have shown at the qualitative level that available data already discriminates between different PDF sets, that is, between different large- $x$ gluon PDFs. Now we will be more quantitative and determine if available data can help in reducing the gluon uncertainties within a single PDF set. In order to quantify this impact, we will use the

\footnotetext{
${ }^{8}$ An alternative way to conveying in a more quantitative way the information contained in figure 4 is to compute the effective $\alpha_{s}$ exponent of the cross section, $n_{\mathrm{eff}} \equiv d \ln \sigma(t \bar{t}) / d \ln \alpha_{s}$, in the two cases, varying the PDF together with $\alpha_{s}$ and keeping the PDFs fixed and varying only $\alpha_{s}$ in the matrix element. At LHC $7 \mathrm{TeV}$, using NNPDF2.3 we find $n_{\mathrm{eff}} \sim 2.7$ in the former and $n_{\mathrm{eff}} \sim 2.3$ in the latter, confirming the effect of the positive correlation between the gluon and $\alpha_{s}$ in the top quark production region.
} 

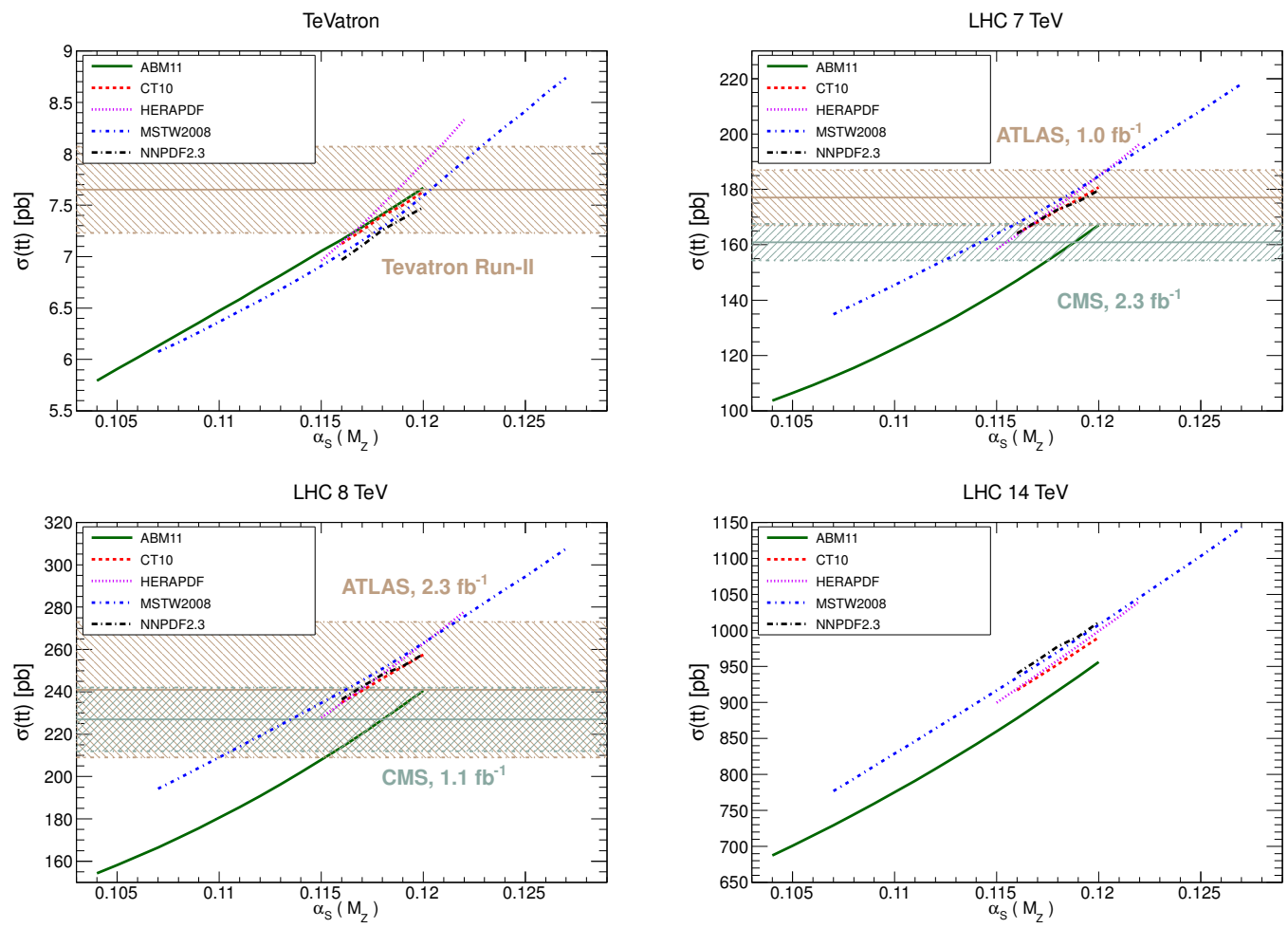

Figure 3. The theoretical predictions for the various PDF sets studied in this work as a function of the strong coupling constant $\alpha_{s}\left(M_{Z}\right)$, compared to the best available experimental data. From top to bottom we show Tevatron, LHC $7 \mathrm{TeV}, 8 \mathrm{TeV}$ and $14 \mathrm{TeV}$.
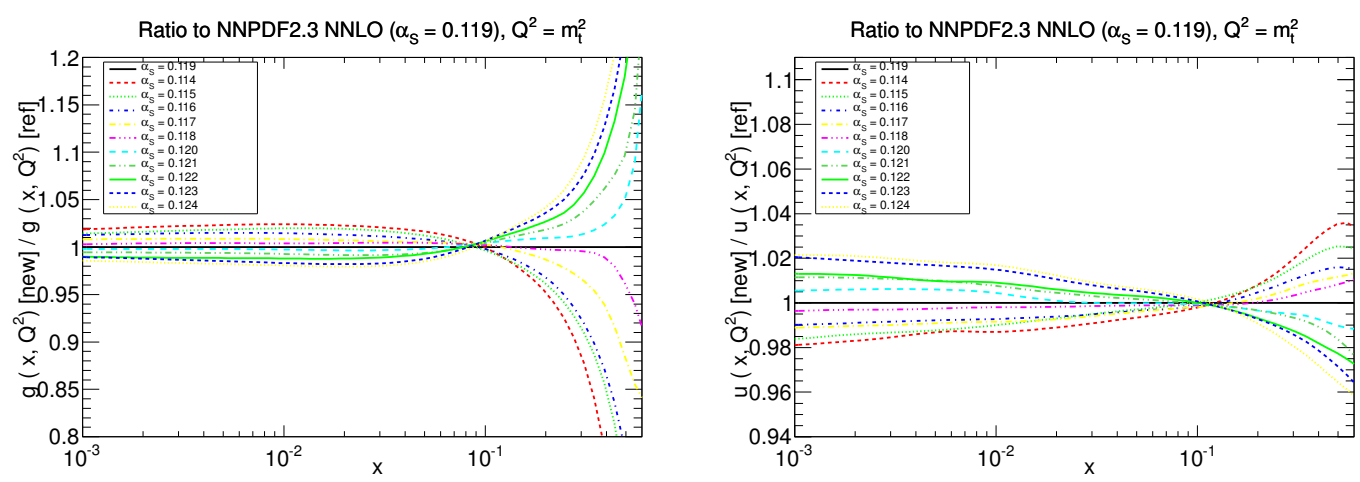

Figure 4. Left plot: the gluon PDF in the NNPDF2.3 fits obtained for different input values of $\alpha_{s}\left(M_{Z}\right)$, evaluated at $Q^{2}=m_{t}^{2}$, shown as the ratio with respect to the gluon PDF obtained for $\alpha_{s}\left(M_{Z}\right)=0.119$. Right plot: the same comparison, this time for the up quark PDF.

Bayesian PDF reweighting method of refs. [71, 72] on the NNPDF2.3 set. ${ }^{9}$ The same study could be carried out with Hessian PDF sets as discussed in [74]. In principle, one could also use the top quark differential distributions data from ATLAS and CMS [75, 76]. However,

\footnotetext{
${ }^{9}$ An alternative possibility would have been a direct inclusion of the top quark data in the NNPDF framework using MCFM code together with the fast interface for $t \bar{t}$ production provided by APPLgrid [73]. This fast interface will be used in future NNPDF releases which will include relevant top quark production data.
} 

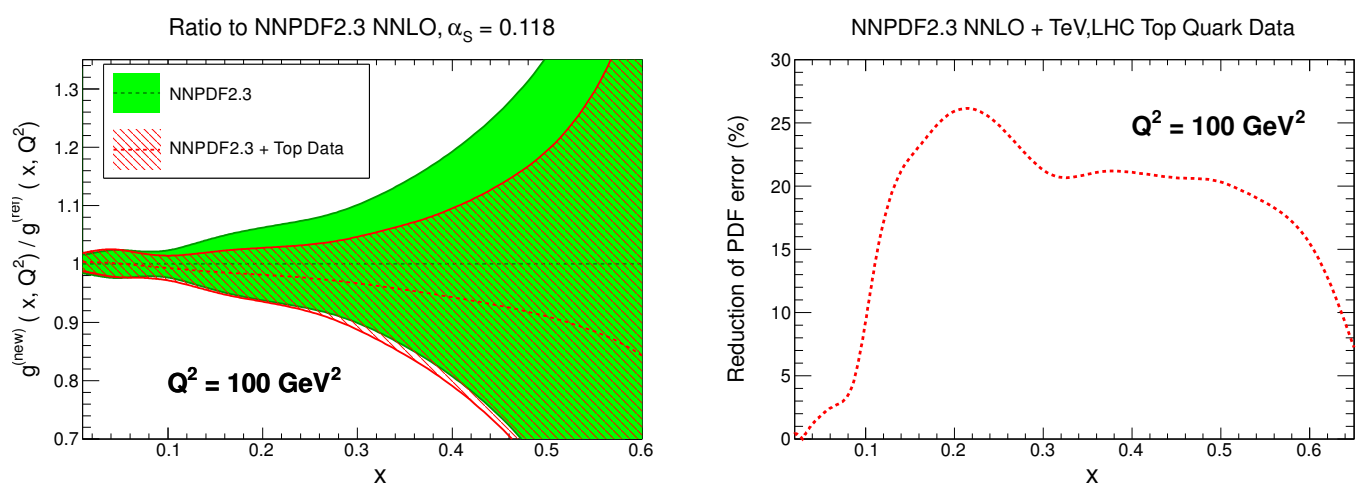

Figure 5. Left plot: the ratio of the NNPDF2.3 NNLO gluon PDF at $Q^{2}=100 \mathrm{GeV}^{2}$ between the default fit and after including the Tevatron and LHC top quark cross section data. Right plot: the relative reduction of PDF uncertainties thanks to the inclusion of top data in the PDF fit.

these data are less precise than the total cross section measurement and the corresponding theoretical predictions are currently only available at NLO. ${ }^{10}$

Therefore, we have included the $N_{\text {dat }}=5$ experimental data points available from the Tevatron and the LHC into the NNPDF2.3 NNLO fit. The definition of $\chi^{2}$ that we use is eq. (3.1). The effective number of replicas after reweighting (the exponential of the Shannon entropy) is $N_{\text {eff }}=86$, out of the starting sample of 100 replicas, indicating the moderate constraining power of the data.

The results of adding the top quark data into the gluon PDF are shown in figure 5 . We show the NNPDF2.3 NNLO gluon at $Q^{2}=100 \mathrm{GeV}^{2}$, in the default fit and after including the Tevatron and LHC top quark production cross section data. We observe that the large- $x$ gluon PDF uncertainties decrease. This is expected since in that region the correlation between the gluon and the top-quark cross section is maximal (see figure 1). In figure 5 we also show the relative reduction in PDF uncertainties from the addition of top data. This reduction coincides, within the finite statistics of the original NNPDF Monte Carlo sample, with the correlation profile of figure 1, and in particular confirms that the top quark data have a small impact below $x \sim 0.1$ or so, as well as for very large values of $x$. Therefore, we conclude that the available top data can already help reduce the uncertainties on the gluon PDF by a factor of up to $20 \%$, and are thus an important ingredient to future global PDF analyses. On the other hand, the impact of top data on the quark PDFs is essentially negligible.

It is interesting to study the modifications of the theory predictions after the top quark data have been added into the NNPDF2.3 fit. In table 9 we show the $t \bar{t}$ cross section for NNPDF2.3, comparing the default prediction with the predictions after adding different subsets of the top quark data. We show only the entries which correspond to pure predictions. By including top data from lower energy colliders, we can provide arguably the most accurate theoretical prediction for the total $t \bar{t}$ cross section at higher energies,

\footnotetext{
${ }^{10}$ For some specific differential distributions, results at NLO supplemented by threshold resummation are available, see $[9,77]$ and references therein.
} 


\begin{tabular}{|c|c|c|c|c|}
\hline Collider & Ref. & Ref $+\mathrm{TeV}$ & Ref. $+\mathrm{TeV}+\mathrm{LHC} 7$ & Ref+TeV+LHC7+8 \\
\hline Tevatron & $7.26 \pm 0.12$ & $(7.29 \pm 0.12)$ & $(7.27 \pm 0.12)$ & $(7.27 \pm 0.12)$ \\
\hline LHC $7 \mathrm{TeV}$ & $172.5 \pm 5.2$ & $172.7 \pm 5.1$ & $(170.5 \pm 3.6)$ & $(170.5 \pm 3.5)$ \\
LHC $8 \mathrm{TeV}$ & $247.8 \pm 6.6$ & $248.0 \pm 6.5$ & $245.0 \pm 4.6$ & $(245.2 \pm 4.4)$ \\
LHC $14 \mathrm{TeV}$ & $976.5 \pm 16.4$ & $976.2 \pm 16.3$ & $969.8 \pm 12.0$ & $969.6 \pm 11.6$ \\
\hline
\end{tabular}

Table 9. The $t \bar{t}$ cross section $\sigma_{t \bar{t}}$, in picobarns, for the NNPDF2.3 NNLO set, together with the associated PDF uncertainties. We show both the reference predictions with NNPDF2.3, and the predictions once Tevatron, LHC7 and LHC8 data are added sequentially to the fitted dataset. We show both the predictions corresponding to beam energies whose data have not been included in the fit, and the post-dictions (in parenthesis and italics) for the beam energies whose data has been used in the fit. The fourth column corresponds to the best theory prediction for LHC $8 \mathrm{TeV}$, while the last column is the best theory prediction for LHC $14 \mathrm{TeV}$.

\begin{tabular}{|c|c|c|c|}
\hline Collider & NNPDF2.3 & $\begin{array}{c}\text { NNPDF2.3 } \\
+ \text { TeV }, \text { LHC data }\end{array}$ & $\begin{array}{c}\text { NNPDF2.3 } \\
+\mathrm{TeV}, \text { LHC 7 TeV data }\end{array}$ \\
\hline$\chi^{2}\left(\right.$ Total,$\left.N_{\text {dat }}=5\right)$ & 6.28 & 4.88 & 4.87 \\
$\chi^{2}\left(\mathrm{LHC} 8 \mathrm{TeV}, N_{\text {dat }}=2\right)$ & 1.64 & 1.24 & 1.24 \\
\hline
\end{tabular}

Table 10. The NNPDF2.3 $\chi^{2}$ compared to the hadron collider top quark production data, before adding any data (first column), after adding all Tevatron and LHC data (second column) and adding only the Tevatron and LHC $7 \mathrm{TeV}$ data points (third column). Note that the $\chi^{2}$ is not normalized by the number of data points.

given that PDF uncertainties will be reduced in the same kinematical range from lower energy data. ${ }^{11}$

These predictions are collected in table 9. As an illustration, the NNPDF2.3 prediction including Tevatron and LHC 7 top data would be the best available theory prediction for LHC $8 \mathrm{TeV}$. Note that not only PDF uncertainties are reduced, but that also the central value is shifted to improve the agreement with the experimental data. As can be seen, the precise $7 \mathrm{TeV}$ data carry most of the constraining power, though of course improved power of the $8 \mathrm{TeV}$ data will be provided with the analysis of the full 2012 dataset.

Then in table 10 we provide NNPDF2.3 $\chi^{2}$ compared to the top quark data, before adding any data, after adding all Tevatron and LHC data and adding only the Tevatron and $\mathrm{LHC} 7 \mathrm{TeV}$ data points. The slight improvement of an already good quantitative description can be seen. As expected, the agreement of the prediction with LHC8 data, when only Tevatron and LHC7 data are used, is a non-trivial consistency check of the whole procedure. ${ }^{12}$

\footnotetext{
${ }^{11}$ Note that, as shown by figure 1 , the typical $x$ ranges covered by the theory predictions at LHC 7,8 and $14 \mathrm{TeV}$ are quite similar, justifying the extrapolation of lower LHC energy data to improve the theory predictions at higher LHC center of mass energies.

${ }^{12}$ The small change of the $\chi^{2}$ between TEV +LHC data and TEV+LHC7 data is due to statistical fluctuations, reflecting the fact that the $8 \mathrm{TeV}$ data are still not precise enough to provide constraints on the gluon PDF.
} 

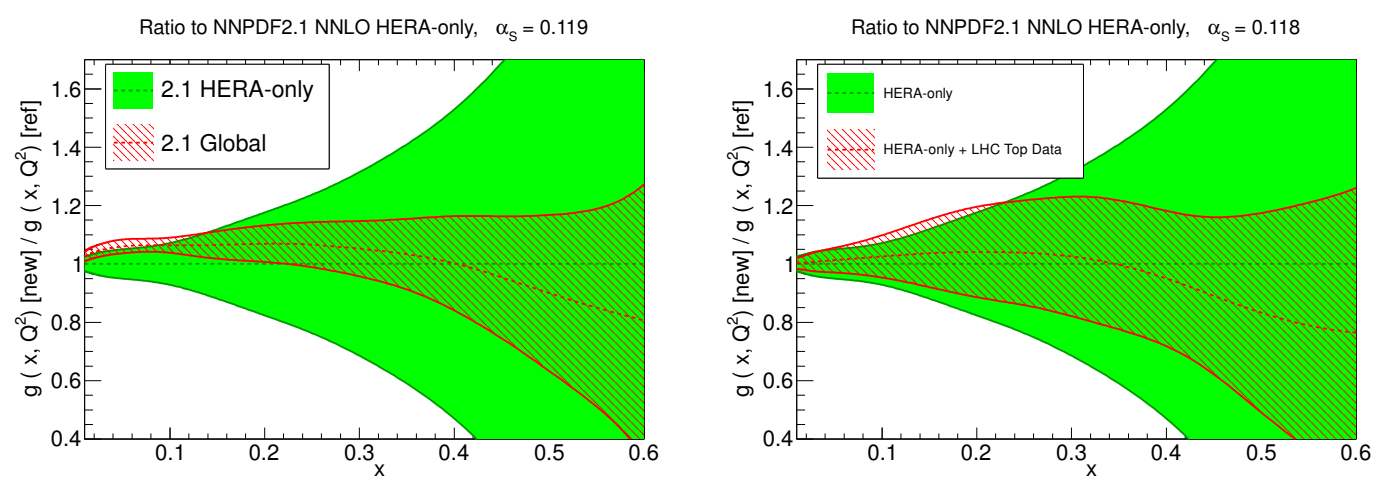

Figure 6. Left plot: the NNPDF2.1 HERA-only gluon PDF, at $Q^{2}=100 \mathrm{GeV}^{2}$, compared to the reference global NNPDF2.1 gluon. Right plot: the NNPDF2.1 HERA-only gluon before and after including the LHC top quark production cross sections.

Given that the constraints from top quark data in a global PDF fit such as NNPDF2.3 are already substantial, we expect even larger constrains in PDF sets based on reduced datasets. To quantify the impact of top data into a DIS-only PDF fit, we have performed a similar analysis as the one with NNPDF2.3, but now starting from a PDF set based on a reduced dataset, the NNPDF2.1 HERA-only set [78, 79]. As the name indicates, this PDF set includes only HERA data, and thus is affected by larger PDF uncertainties than the NNPDF global fits, in particular for the large- $x$ gluon.

In figure 6 we compare first of all the gluon from the NNPDF2.1 HERA-only fit with that of the NNPDF2.1 global fit, to show the large differences in PDF uncertainties due to the reduced dataset in the former case. Then we show the improvements in the gluon PDF in the HERA-only fit after the addition of the top quark data. It is clear that the impact is substantial. Remarkably, the top quark data bring the gluon from the HERA-only fit closer to the gluon from the global fit.

\subsection{Impact on predictions for BSM particle production}

Many scenarios of BSM physics predict the production of massive final states in gluoninitiated processes. The improvement in the large- $x$ gluon PDF uncertainties seen in figure 5 implies a similar improvement in any cross section that is dominated by initial-state gluons in a similar kinematical region. Production kinematics determines that any highmass final state that is gluon initiated will benefit from the improvement in PDF uncertainties. Therefore, now we explore in two cases the phenomenological implications of the improved large- $x$ gluon: the production of heavy Kaluza-Klein resonances in warped extra dimensions scenarios, and the PDF uncertainties in the high invariant mass distribution of top quark pairs.

To begin with, we have considered Kaluza-Klein massive graviton production in warped extra dimension scenarios [20], in particular in the so-called bulk models in which the coupling of the graviton to fermions is suppressed, and thus production is driven by gluongluon annihilation [21]. We have computed the cross sections for Randall-Sundrum graviton production at the LHC $8 \mathrm{TeV}$ at leading order using the MadGraph5 program [80], for a range 


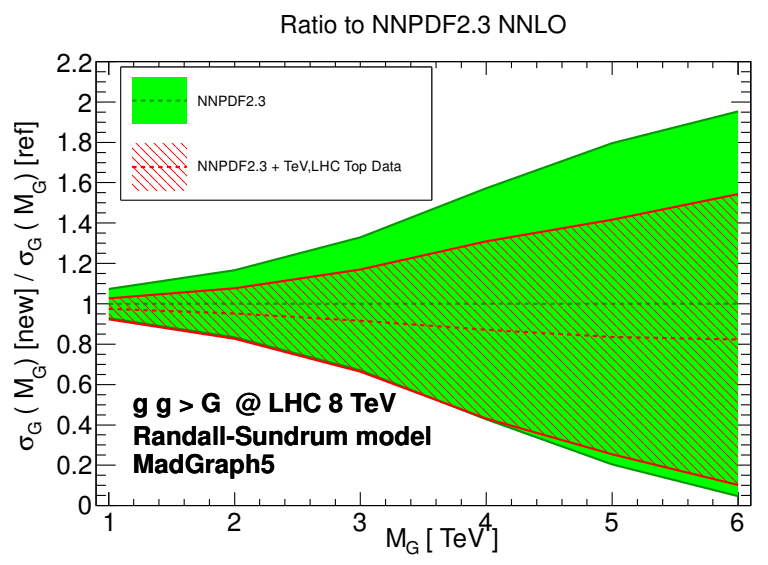

Figure 7. The PDF uncertainties for the production of Randall-Sundrum Kaluza-Klein gravitons at the LHC $8 \mathrm{TeV}$, with NNPDF2.3 before and after including the top quark data, as a function of the graviton mass $M_{G}$. We have assumed that the graviton couples only to gluons. The cross section has been computed at leading order with MadGraph5.

of values of the graviton mass $M_{G}$. The improvement in theoretical (PDF) uncertainties in the graviton production cross section thanks to top quark data is shown in figure 7 . We see that, to begin with, PDF uncertainties are large, almost $40 \%$ for $M_{G}=3 \mathrm{TeV}$, and growing as we approach the kinematic threshold, reflecting our lack of knowledge of the large- $x$ gluon PDF. The top quark data reduce moderately the production uncertainties. Of course, future, more precise top data will render these constraints more stringent, which in turn translate into the possibility of better characterizing eventual BSM high mass particles that the LHC could find. Similar improvements are found for $14 \mathrm{TeV}$.

The second example is the high mass tail of the invariant mass distribution in topquark production, relevant for many BSM searches [22, 23], which is also related with the top quark forward-backward asymmetry measured at the Tevatron. One example is the searches for heavy resonances that decay into a top-antitop pair [81-84]. To study the impact of the improvement of gluon PDF uncertainties there, we have computed top quark pair production with the aMC@NLO program [85-87], at NLO matched to the Herwig6 shower [88]. The renormalization and factorization scales are set equal to the sum of transverse masses of all final-state particles. Using NNPDF2.3, we have evaluated the cross section with a cut in the minimum invariant mass of the $t \bar{t}$ pair, $M_{t t}$, before and after including the top quark data into the PDF fit. We show the results in figure 8, for the absolute cross section (left) and for the relative scale and PDF uncertainties (right) as a function of the minimum value of $M_{t t}$ allowed.

As we can see, the addition of the total $t \bar{t}$ cross section data reduces the PDF uncertainty in the high-end tail of the $t \bar{t}$ mass distribution. It must be remarked that the data in this high-mass tail are only a negligible fraction of the total $t \bar{t}$ cross section, and therefore do not play any role in the PDF fit itself. The PDF fit including $\sigma(t \bar{t})$ reduces the gluon uncertainty at large $x$ only because of the overall constraints on the PDF evolution, which correlate the $x$ behavior in the $x \sim 0.1$ region (which dominates the total production cross section) and the large- $x$ region, which is relevant to the high-mass behavior. 

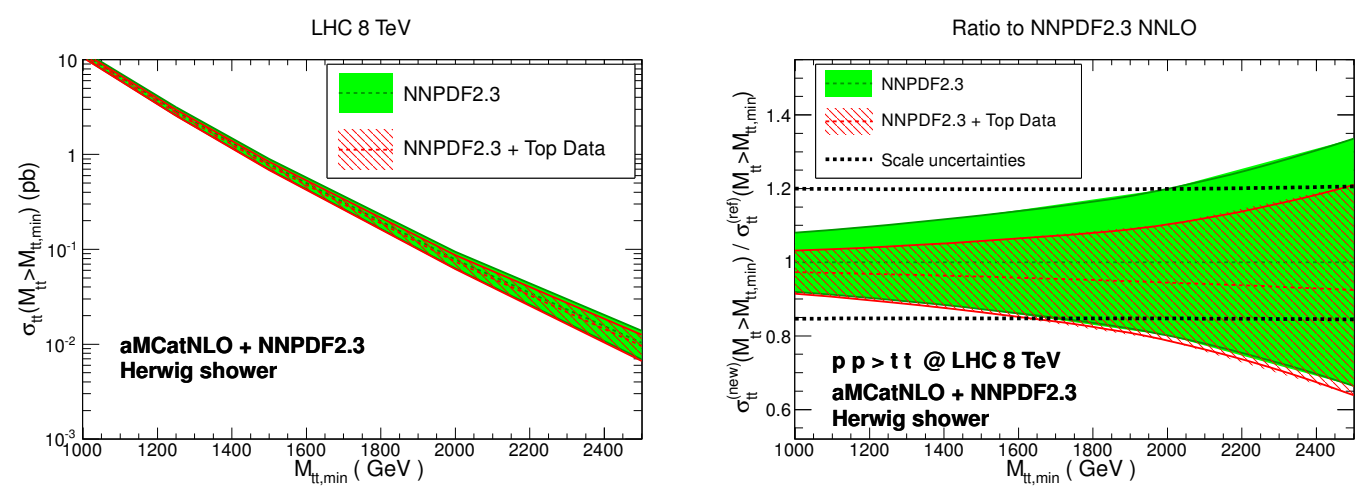

Figure 8. Left plot: the $t \bar{t}$ cross section above a minimum value of the $t \bar{t}$ invariant mass $M_{t t}$, computed with aMCatNLO with the Herwig parton shower, and the NNPDF2.3 as input set, at the LHC $8 \mathrm{TeV}$. The error band is the PDF uncertainty band. Right plot: the relative scale and PDF uncertainties for the cross section of top quark pairs with invariant mass above $M_{t t, \min }$, at LHC $8 \mathrm{TeV}$. In both cases we show the predictions with NNPDF2.3 before and after including the top quark data into the PDF fit.

As in the case of dijet cross sections, we expect that rate measurements in kinematical regions where, for example, the $t \bar{t}$ system has a large rapidity, can be used to further improve the knowledge of large- $x$ gluons, and improve even more the precision of predictions for the production of large-mass objects in $g g$-initiated channels.

\section{Cross-section ratios between different LHC beam energies}

The measurement of cross-section ratios between different center of mass energies at the LHC has two main motivations [89]: first, they are interesting for precision SM studies and second, they have the potential to enhance the possible BSM sensitivity of absolute cross sections. In ref. [89], results for top quark cross sections based on NLO+NNLL theory were provided; here we update them to NNLO+NNLL and compare them with experimental data.

First of all we show the correlations between PDFs and the cross-section ratios in figure 9. We see that the PDF correlation is approximately the inverse of that of the absolute cross sections, shown in figure 1 . The reason for this anti-correlation at large $x$ is that when going to higher energies, the average probed values of $x$ are smaller.

Using the same settings as before, we now provide NNLO+NNLL predictions for the cross-section ratios in tables 11 and 12 . We note that all systematic theoretical uncertainties are small: in the ratio between 8 and $7 \mathrm{TeV}$ the total theory uncertainty is at the permille level, while in the ratio between 14 and $8 \mathrm{TeV}$, the total error on the ratio is at most around $2 \%$. This is dominated by PDF uncertainty, which is four times larger than the combined scale, $\alpha_{s}$ and $m_{t}$ uncertainties. Notice the great improvement due to the NNLO result: in the NLO+NNLL analysis of top cross-section ratios of ref. [89], scale and PDF uncertainties were of similar size. We note that, as can be seen from table 12, in the case of the 14 over $8 \mathrm{TeV}$ ratios the differences between PDF sets are at the level of $10 \%$. 

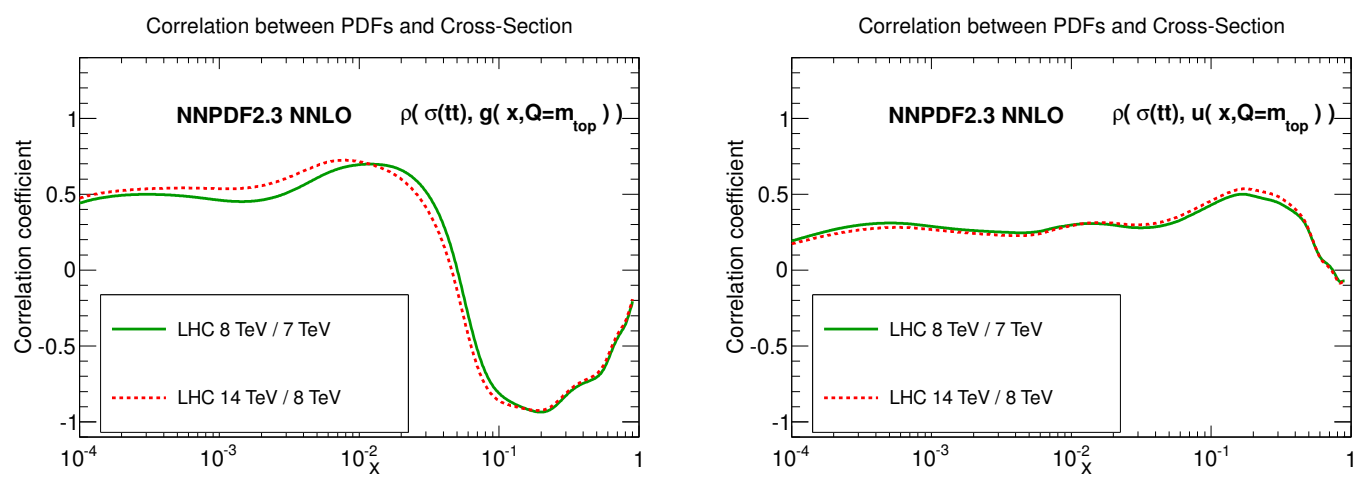

Figure 9. The correlation between the gluon PDF (left plot) and the up quark PDF (right plot) with the top quark production cross-section ratios between different LHC center of mass energies. The correlations are computed for $Q=m_{t}=173.3 \mathrm{GeV}$.

\begin{tabular}{|c|c|c|c|c|c|c|}
\hline \multicolumn{7}{|c|}{ LHC $8 \mathrm{TeV} / 7 \mathrm{TeV}$ ratio } \\
\hline PDF set & $\sigma_{t t}$ & $\delta_{\text {scale }}$ & $\delta_{\mathrm{PDF}}$ & $\delta_{\alpha_{s}}(\mathrm{pb})$ & $\delta_{\mathrm{m}_{\mathrm{t}}}$ & $\delta_{\text {tot }}$ \\
\hline ABM11 & 1.463 & $\begin{array}{ll}+0.001 & (+0.1 \%) \\
-0.002 & (-0.1 \%)\end{array}$ & $\begin{array}{ll}+0.006 & (+0.4 \%) \\
-0.006 & (-0.4 \%)\end{array}$ & $\begin{array}{ll}+0.000 & (+0.0 \%) \\
-0.000 & (-0.0 \%)\end{array}$ & $\begin{array}{ll}+0.001 & (+0.1 \%) \\
-0.001 & (-0.1 \%)\end{array}$ & $\begin{array}{ll}+0.007 & (+0.5 \%) \\
-0.008 & (-0.5 \%)\end{array}$ \\
\hline CT10 & 1.428 & $\begin{array}{ll}+0.001 & (+0.1 \%) \\
-0.001 & (-0.1 \%)\end{array}$ & $\begin{array}{ll}+0.008 & (+0.5 \%) \\
-0.010 & (-0.7 \%)\end{array}$ & $\begin{array}{ll}+0.002 & (+0.2 \%) \\
-0.002 & (-0.2 \%)\end{array}$ & $\begin{array}{ll}+0.001 & (+0.1 \%) \\
-0.001 & (-0.1 \%)\end{array}$ & $\begin{array}{ll}+0.009 & (+0.6 \%) \\
-0.011 & (-0.8 \%)\end{array}$ \\
\hline HERA1.5 & 1.426 & $\begin{array}{ll}+0.001 & (+0.0 \%) \\
-0.002 & (-0.1 \%)\end{array}$ & $\begin{array}{ll}+0.003 & (+0.2 \%) \\
-0.003 & (-0.2 \%)\end{array}$ & $\begin{array}{ll}+0.001 & (+0.1 \%) \\
-0.001 & (-0.1 \%)\end{array}$ & $\begin{array}{ll}+0.001 & (+0.1 \%) \\
-0.001 & (-0.1 \%)\end{array}$ & $\begin{array}{ll}+0.004 & (+0.3 \%) \\
-0.005 & (-0.4 \%)\end{array}$ \\
\hline JR09 & 1.426 & $\begin{array}{ll}+0.001 & (+0.0 \%) \\
-0.001 & (-0.0 \%)\end{array}$ & $\begin{array}{ll}+0.014 & (+1.0 \%) \\
-0.014 & (-1.0 \%)\end{array}$ & $\begin{array}{ll}+0.000 & (+0.0 \%) \\
-0.000 & (-0.0 \%)\end{array}$ & $\begin{array}{ll}+0.001 & (+0.1 \%) \\
-0.001 & (-0.1 \%)\end{array}$ & $\begin{array}{ll}+0.014 & (+1.0 \%) \\
-0.014 & (-1.0 \%)\end{array}$ \\
\hline MSTW08 & 1.429 & $\begin{array}{ll}+0.001 & (+0.1 \%) \\
-0.001 & (-0.1 \%)\end{array}$ & $\begin{array}{ll}+0.004 & (+0.2 \%) \\
-0.004 & (-0.2 \%)\end{array}$ & $\begin{array}{ll}+0.001 & (+0.1 \%) \\
-0.001 & (-0.1 \%)\end{array}$ & $\begin{array}{ll}+0.001 & (+0.1 \%) \\
-0.001 & (-0.1 \%)\end{array}$ & $\begin{array}{ll}+0.005 & (+0.3 \%) \\
-0.005 & (-0.3 \%)\end{array}$ \\
\hline NNPDF2.3 & 1.437 & $\begin{array}{rr}+0.001 & (+0.1 \%) \\
-0.001 & (-0.1 \%) \\
\end{array}$ & $\begin{array}{rr}+0.006 & (+0.4 \%) \\
-0.006 & (-0.4 \%) \\
\end{array}$ & $\begin{array}{cc}+0.001 & (+0.1 \%) \\
-0.001 & (-0.1 \%) \\
\end{array}$ & $\begin{array}{cc}+0.001 & (+0.1 \%) \\
-0.001 & (-0.1 \%) \\
\end{array}$ & $\begin{array}{ll}+0.007 & (+0.5 \%) \\
-0.007 & (-0.5 \%) \\
\end{array}$ \\
\hline ATLAS & 1.36 & & & & & $\pm 0.11(8 \%)$ \\
\hline CMS & 1.40 & & & & & $\pm 0.08(6 \%)$ \\
\hline
\end{tabular}

Table 11. The NNLO+NNLL predictions for the ratio of top quark cross section at the LHC between 8 and $7 \mathrm{TeV}$, with all the PDFs considered, and with the various sources of theoretical uncertainties. The default value of $\alpha_{s}\left(M_{Z}\right)$ from each collaboration has been used in the computation of the central predictions. The lower row shows our estimate of the best available experimental measurement. The total theoretical uncertainty is the linear sum of scale and parametric uncertainties, as discussed in the text.

Therefore, such measurement would provide powerful PDF constraints even within the foreseeable experimental accuracies.

We have checked that the results computed at NNLO+NNLL with NNPDF2.1 are fully consistent with the computation of ref. [89], performed at NLO+NNLL accuracy. This proves the reliability of the theoretical uncertainty on the cross section ratios for $t \bar{t}$ production presented in [89], and underscores the stability of this ratio under higherorder corrections. Indeed, central values and PDF uncertainties are unchanged, while scale uncertainties are further decreased. For instance, at NLO+NNLL the scale uncertainty in the $14 / 8$ ratio was about $1 \%$, while with the NNLO+NNLL computation it decreases down to $0.3 \%$.

While no measurement of the ratio between 8 and $7 \mathrm{TeV}$ is available, we can estimate the expected precision from the absolute cross-section measurements of table 2 by assuming 


\begin{tabular}{|c|c|c|c|c|c|c|}
\hline \multicolumn{7}{|c|}{ LHC $14 \mathrm{TeV} / 8 \mathrm{TeV}$ ratio } \\
\hline PDF set & $\sigma_{t t}$ & $\delta_{\text {scale }}$ & $\delta_{\mathrm{PDF}}$ & $\delta_{\alpha_{s}}(\mathrm{pb})$ & $\delta_{\mathrm{m}_{\mathrm{t}}}$ & $\delta_{\text {tot }}$ \\
\hline ABM11 & 4.189 & $\begin{array}{ll}+0.008 & (+0.2 \%) \\
-0.016 & (-0.4 \%)\end{array}$ & $\begin{array}{ll}+0.057 & (+1.4 \%) \\
-0.057 & (-1.4 \%)\end{array}$ & $\begin{array}{ll}+0.000 & (+0.0 \%) \\
-0.000 & (-0.0 \%)\end{array}$ & $\begin{array}{ll}+0.012 & (+0.3 \%) \\
-0.012 & (-0.3 \%)\end{array}$ & $\begin{array}{ll}+0.067 & (+1.6 \%) \\
-0.074 & (-1.8 \%)\end{array}$ \\
\hline CT10 & 3.869 & $\begin{array}{ll}+0.006 & (+0.2 \%) \\
-0.009 & (-0.2 \%)\end{array}$ & $\begin{array}{ll}+0.068 & (+1.8 \%) \\
-0.088 & (-2.3 \%)\end{array}$ & $\begin{array}{ll}+0.020 & (+0.5 \%) \\
-0.020 & (-0.5 \%)\end{array}$ & $\begin{array}{ll}+0.010 & (+0.2 \%) \\
-0.010 & (-0.2 \%)\end{array}$ & $\begin{array}{ll}+0.077 & (+2.0 \%) \\
-0.100 & (-2.6 \%)\end{array}$ \\
\hline HERA1.5 & 3.841 & $\begin{array}{ll}+0.005 & (+0.1 \%) \\
-0.012 & (-0.3 \%)\end{array}$ & $\begin{array}{ll}+0.033 & (+0.9 \%) \\
-0.025 & (-0.7 \%)\end{array}$ & $\begin{array}{ll}+0.010 & (+0.3 \%) \\
-0.010 & (-0.3 \%)\end{array}$ & $\begin{array}{ll}+0.009 & (+0.2 \%) \\
-0.010 & (-0.2 \%)\end{array}$ & $\begin{array}{ll}+0.041 & (+1.1 \%) \\
-0.041 & (-1.1 \%)\end{array}$ \\
\hline JR09 & 3.808 & $\begin{array}{ll}+0.005 & (+0.1 \%) \\
-0.005 & (-0.1 \%)\end{array}$ & $\begin{array}{ll}+0.117 & (+3.1 \%) \\
-0.117 & (-3.1 \%)\end{array}$ & $\begin{array}{ll}+0.000 & (+0.0 \%) \\
-0.000 & (-0.0 \%)\end{array}$ & $\begin{array}{ll}+0.012 & (+0.3 \%) \\
-0.012 & (-0.3 \%)\end{array}$ & $\begin{array}{ll}+0.122 & (+3.2 \%) \\
-0.122 & (-3.2 \%)\end{array}$ \\
\hline MSTW08 & 3.880 & $\begin{array}{ll}+0.006 & (+0.2 \%) \\
-0.009 & (-0.2 \%)\end{array}$ & $\begin{array}{ll}+0.036 & (+0.9 \%) \\
-0.036 & (-0.9 \%)\end{array}$ & $\begin{array}{ll}+0.011 & (+0.3 \%) \\
-0.011 & (-0.3 \%)\end{array}$ & $\begin{array}{ll}+0.010 & (+0.2 \%) \\
-0.010 & (-0.2 \%)\end{array}$ & $\begin{array}{ll}+0.045 & (+1.2 \%) \\
-0.048 & (-1.2 \%)\end{array}$ \\
\hline NNPDF2.3 & 3.940 & $\begin{array}{ll}+0.006 & (+0.2 \%) \\
-0.010 & (-0.3 \%)\end{array}$ & $\begin{array}{ll}+0.048 & (+1.2 \%) \\
-0.048 & (-1.2 \%)\end{array}$ & $\begin{array}{ll}+0.009 & (+0.2 \%) \\
-0.009 & (-0.2 \%)\end{array}$ & $\begin{array}{ll}+0.010 & (+0.3 \%) \\
-0.010 & (-0.3 \%)\end{array}$ & $\begin{array}{l}+0.056(+1.4 \%) \\
-0.060(-1.5 \%)\end{array}$ \\
\hline
\end{tabular}

Table 12. Same as table 11 for the ratio of 14 over $8 \mathrm{TeV}$ cross sections at the LHC.

that all systematic uncertainties (but not the luminosity) are fully correlated between 8 and $7 \mathrm{TeV}$. This leads to

$$
\begin{aligned}
& \sigma_{\mathrm{LHC} 8 / 7}^{(\text {Atlas })}(t \bar{t})=1.36 \pm 0.11 \mathrm{pb}(8 \%), \\
& \sigma_{\mathrm{LHC} / 7}^{(\mathrm{CMS})}(t \bar{t})=1.40 \pm 0.08 \mathrm{pb}(6 \%) .
\end{aligned}
$$

Note that while ATLAS is higher than CMS at 8 and $7 \mathrm{TeV}$, this trend is inverted in the cross section ratio.

If no such correlation between the systematic uncertainties is assumed we get

$$
\begin{aligned}
& \sigma_{\mathrm{LHC} 8 / 7}^{(\mathrm{Atlas})}(t \bar{t})=1.36 \pm 0.20 \mathrm{pb}(15 \%), \\
& \sigma_{\mathrm{LHC} 8 / 7}^{(\mathrm{CMS})}(t \bar{t})=1.40 \pm 0.11 \mathrm{pb}(8 \%)
\end{aligned}
$$

which illustrates the importance of maximizing the cancellation of systematics. This requires a dedicated strategy for the ratio measurement, rather than simply combining data at two different energies.

We compare graphically the theoretical predictions for the ratios between 8 and $7 \mathrm{TeV}$ and 14 and $8 \mathrm{TeV}$ in figure 10. In the first case, we also show the estimate of the ATLAS and CMS results, assuming full correlation of the systematic uncertainties. Then we show in figure 11 the dependence on $\alpha_{s}\left(M_{Z}\right)$ of the NNLO+NNLL cross-section ratios for each of the various PDF sets. The slope with $\alpha_{s}$ for the prediction of each of the PDF sets varies more than for the absolute cross sections, figure 3 . However, with the assumed uncertainty on $\alpha_{s}$, the contribution $\delta_{\alpha_{s}}$ to the total theory uncertainty of the cross-section ratios is much smaller than the PDF uncertainty.

Finally, it is useful to provide predictions for the cross section ratios in the case of NNPDF2.3 supplemented by the Tevatron and LHC top data, discussed in the previous section. The results are shown in table 13. As can be seen, while the differences are non negligible, they are probably well beyond foreseeable experimental accuracies. This is expected since cross-section ratios are mostly sensitive to more dramatic differences between PDF sets, such as those between AMB11 and the other sets in the case of the 14 over $8 \mathrm{TeV}$ cross-section ratio, as shown in Fig 10. 

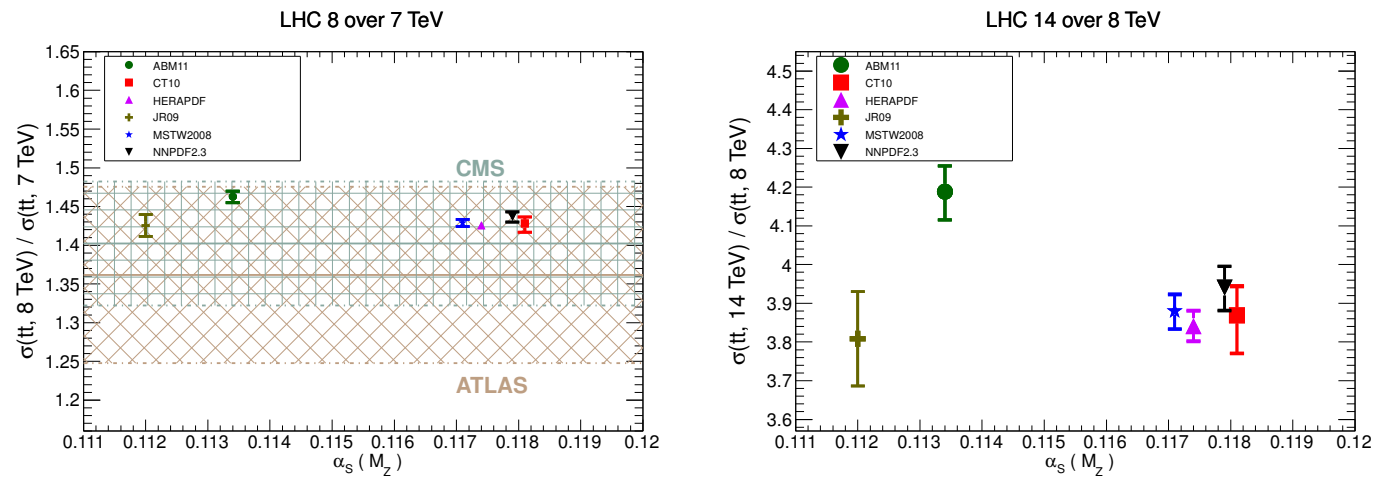

Figure 10. The best predictions from each PDF set for the LHC ratios between 8 and $7 \mathrm{TeV}$ (left plot) and 14 and $8 \mathrm{TeV}$ (right plot). The error bars correspond to the total theoretical uncertainty. For the $8 / 7$ ratio, the ATLAS and CMS experimental results have been obtained taking the ratio of the central data and assuming that experimental systematics (but not luminosity) is fully correlated between different beam energies.
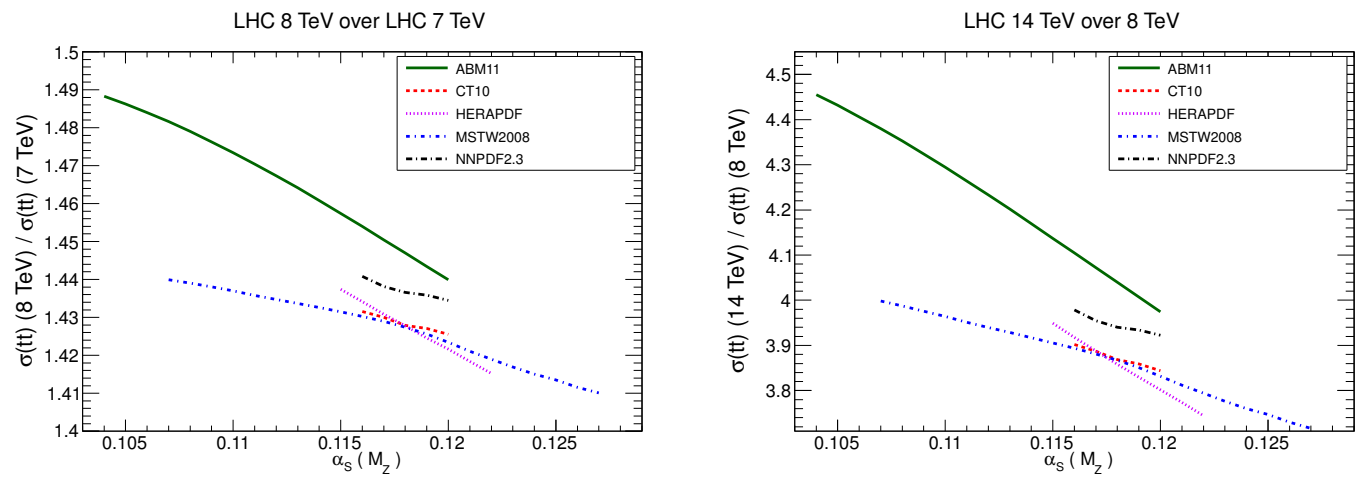

Figure 11. The theoretical predictions for the cross section ratios between different LHC beam energies for the the various PDF sets, as a function of the strong coupling constant $\alpha_{s}\left(M_{Z}\right)$.

\begin{tabular}{|c|cc|c|}
\hline Collider & \multicolumn{2}{|c|}{ NNPDF2.3 } & NNPDF2.3 + TeV,LHC top data \\
\hline$\sigma_{\mathrm{LHC} 8 / 7}(t \bar{t})$ & $1.437 \pm 0.006$ & $(0.4 \%)$ & $1.439 \pm 0.005 \quad(0.3 \%)$ \\
$\sigma_{\mathrm{LHC} 14 / 8}(t \bar{t})$ & $3.94 \pm 0.05 \quad(1.2 \%)$ & $3.96 \pm 0.04 \quad(1.0 \%)$ \\
\hline
\end{tabular}

Table 13. The $t \bar{t}$ cross section ratios for the NNPDF2.3 NNLO set, together with the associated PDF uncertainties, both in the reference fit and when NNPDF2.3 is supplemented by Tevatron and LHC top quark production data.

\section{Hypothetical fourth-generation heavy quark production at the LHC}

Following ref. [8], we provide also the total cross section at NNLO+NNLL accuracy for a pair of hypothetical heavy fourth-generation quarks, belonging to the fundamental representation of SU(3). Such new massive fermions arise naturally in BSM theories with strongly-coupled dynamics. We denote these hypothetical heavy fourth-generation quarks by $T$. Our aim is to assess the scale and PDF uncertainties affecting the QCD contribution to the production of such heavy fermions using the most up-to-date theoretical inputs. We 

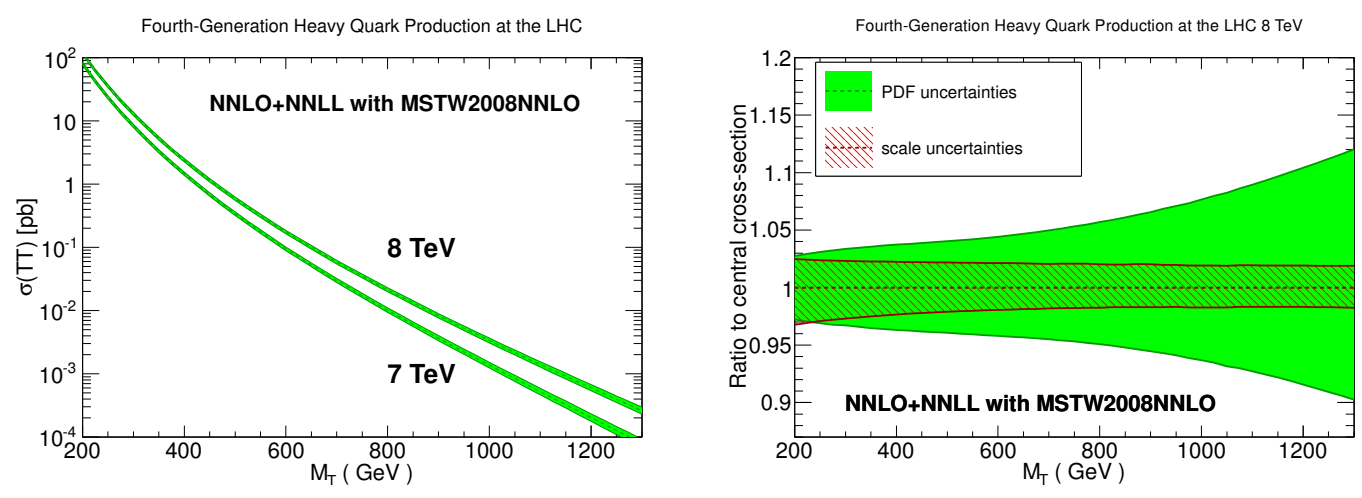

Figure 12. Left plot: the cross sections for the production of a $T \bar{T}$ pair of hypothetical heavy fourth-generation quarks at the LHC 7 and $8 \mathrm{TeV}$, computed at NNLO+NNLL with MSTW2008NNLO, as a function of the heavy quark mass $M_{T}$. The uncertainty band is the linear sum of PDF and scale uncertainties. Right plot: the relative PDF and scale uncertainties as a function of $M_{T}$ for $\mathrm{LHC} 8 \mathrm{TeV}$.

have used both MSTW2008 and NNPDF2.3 NNLO PDFs as input in the computation, and provide predictions for masses $M_{T}$ in the range between $200 \mathrm{GeV}$ and $1.3 \mathrm{TeV}$. The PDF and scale uncertainties are defined as in section 3, with the difference than now the central renormalization and factorization scales are set to the heavy quark mass, $\mu_{F}=\mu_{R}=M_{T}$.

The numerical results for the total cross sections and associated scale and PDF uncertainties have been tabulated and they are available in the source of the arXiv submission of this paper. ${ }^{13}$ In figure 12 (left) we show the production cross sections at LHC 7 and $8 \mathrm{TeV}$ as a function of $M_{T}$, where the uncertainty band is the linear sum of scale and PDF uncertainties. The MSTW08 PDF set was used as input. In figure 12 (right) we also show the relative PDF and scale uncertainties, at the LHC $8 \mathrm{TeV}$. We notice that for large heavy fermion masses $M_{T} \gg m_{t}$, PDF uncertainties become the dominant source of theoretical error.

Another useful comparison is provided by the PDF dependence of the hypothetical heavy fourth-generation quark production cross section. In figure 13 we compare, as a function of $M_{T}$, the predictions for the NNLO+NNLL cross-sections in MSTW08 and NNPDF2.3. Only PDF uncertainties are shown. While the two uncertainty bands overlap, the envelope of the two sets is substantially larger than the bands of the individual sets. The NNPDF2.3 predictions are smaller than the MSTW08 ones, by about 1-sigma, for $M_{T} \geq 400 \mathrm{GeV}$.

\section{Summary and outlook}

In this work we have presented a detailed assessment of the theoretical systematic uncertainties that affect the total top quark pair production cross section at hadron colliders. We have also compared the theoretical predictions with the most recent experimental data from the Tevatron and the LHC 7 and $8 \mathrm{TeV}$, and provided predictions for LHC $14 \mathrm{TeV}$.

\footnotetext{
${ }^{13}$ The data files are heavyfermion_7tev_mstw08.data, heavyfermion_8tev_mstw08.data, heavyfermion _7tev_nnpdf23.data and heavyfermion_7tev_nnpdf23.data.
} 


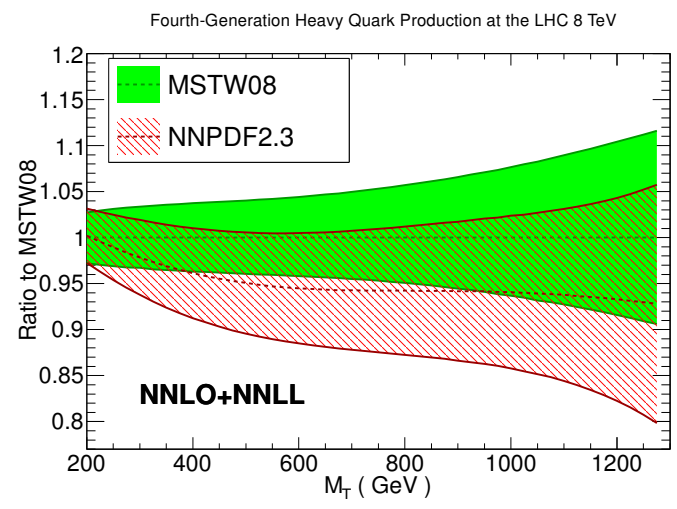

Figure 13. Same as figure 12 (right), now comparing the predictions of MSTW08 and NNPDF2.3. Only PDF uncertainties are shown.

For our analysis we have used the most precise perturbative and non-perturbative information available, in particular the recently computed NNLO+NNLL cross sections and the most up-to-date NNLO PDF sets. Thanks to the significantly reduced scale dependence of the full NNLO calculation, now the three main sources of theoretical uncertainty, scales, PDFs and the top quark mass, are roughly of the same size, around 2-3\%. We find that most PDF sets agree both in their central values and in the size of their PDF uncertainties.

Given the high accuracy of the perturbative $t \bar{t}$ cross section, we have demonstrated that the available data provide a strong constraint on existing NNLO PDF sets, in particular on the large- $x$ gluon PDF. The inclusion of Tevatron and LHC top quark data in the NNPDF2.3 set results in a reduction on the large- $x$ gluon PDF uncertainty, which has further important phenomenological consequences. We have explicitly studied two such cases, both relevant for BSM searches: high mass graviton production in warped extra dimensions scenarios, and the high mass tail of the top pair invariant mass distribution. We have also given updated predictions for the pair production of a hypothetical fourthgeneration heavy quark $T$, which should be helpful in interpreting the results of ongoing experimental searches [90-92].

For completeness, we have also provided up-to-date predictions for the cross-section ratios of $\sigma_{t \bar{t}}$ at different LHC center-of-mass energies.

To conclude, we emphasize that the availability of the NNLO calculation for the total $t \bar{t}$ cross section makes top pair production the first hadron collider process which is both directly sensitive to the gluon PDF and can be included consistently into a NNLO global PDF analysis without any approximation. This leads us to believe that top quark data will be an important ingredient of future PDF fits, especially as more precise data on the total cross section and on various differential distributions becomes available.

\section{Acknowledgments}

We acknowledge Tancredi Carli, Maria José Costa, Roberto Tenchini and Roberto Chierici for information about the LHC top quark cross-section data, and Stefano Forte, Stefano 
Frixione, Katerina Lipka and Sebastian Naumann-Emme for useful discussions. The work of M. C. was supported by the Heisenberg and by the Gottfried Wilhelm Leibniz programmes of the Deutsche Forschungsgemeinschaft, and by the DFG Sonderforschungsbereich/Transregio 9 "Computergestützte Theoretische Teilchenphysik". J. R. is supported by a Marie Curie Intra-European Fellowship of the European Community's 7th Framework Programme under contract number PIEF-GA-2010-272515. The work of M. L. M. and A. M. is supported by the ERC grant 291377 "LHCtheory: Theoretical predictions and analyses of LHC physics: advancing the precision frontier".

Open Access. This article is distributed under the terms of the Creative Commons Attribution License which permits any use, distribution and reproduction in any medium, provided the original author(s) and source are credited.

\section{References}

[1] M. Czakon, P. Fiedler and A. Mitov, The total top quark pair production cross-section at hadron colliders through $O\left(\alpha_{S}^{4}\right)$, arXiv: 1303.6254 [INSPIRE].

[2] P. Baernreuther, M. Czakon and A. Mitov, Percent level precision physics at the Tevatron: first genuine NNLO QCD corrections to $q \bar{q} \rightarrow t \bar{t}+X$, Phys. Rev. Lett. 109 (2012) 132001 [arXiv: 1204.5201] [INSPIRE].

[3] M. Czakon and A. Mitov, NNLO corrections to top-pair production at hadron colliders: the all-fermionic scattering channels, JHEP 12 (2012) 054 [arXiv:1207.0236] [INSPIRE].

[4] M. Czakon and A. Mitov, NNLO corrections to top pair production at hadron colliders: the quark-gluon reaction, JHEP 01 (2013) 080 [arXiv:1210.6832] [INSPIRE].

[5] M. Beneke, P. Falgari and C. Schwinn, Soft radiation in heavy-particle pair production: all-order colour structure and two-loop anomalous dimension, Nucl. Phys. B 828 (2010) 69 [arXiv: 0907.1443] [INSPIRE].

[6] M. Czakon, A. Mitov and G.F. Sterman, Threshold resummation for top-pair hadroproduction to next-to-next-to-leading log, Phys. Rev. D 80 (2009) 074017 [arXiv:0907.1790] [INSPIRE].

[7] M. Cacciari, M. Czakon, M. Mangano, A. Mitov and P. Nason, Top-pair production at hadron colliders with next-to-next-to-leading logarithmic soft-gluon resummation, Phys. Lett. B 710 (2012) 612 [arXiv:1111.5869] [INSPIRE].

[8] M. Cacciari, S. Frixione, M.L. Mangano, P. Nason and G. Ridolfi, Updated predictions for the total production cross sections of top and of heavier quark pairs at the Tevatron and at the LHC, JHEP 09 (2008) 127 [arXiv:0804 .2800] [INSPIRE].

[9] N. Kidonakis and B.D. Pecjak, Top-quark production and QCD, Eur. Phys. J. C 72 (2012) 2084 [arXiv:1108.6063] [INSPIRE].

[10] S. Forte and G. Watt, Progress in the determination of the partonic structure of the proton, arXiv: 1301.6754 [INSPIRE].

[11] G. Watt, Parton distribution function dependence of benchmark standard model total cross sections at the $7 \mathrm{TeV}$ LHC, JHEP 09 (2011) 069 [arXiv:1106.5788] [INSPIRE]. 
[12] G. Watt, MSTW PDFs and impact of PDFs on cross sections at Tevatron and LHC, Nucl. Phys. Proc. Suppl. 222-224 (2012) 61 [arXiv:1201.1295] [INSPIRE].

[13] S. Moch, P. Uwer and A. Vogt, On top-pair hadro-production at next-to-next-to-leading order, Phys. Lett. B 714 (2012) 48 [arXiv:1203.6282] [INSPIRE].

[14] M. Aliev et al., HATHOR: HAdronic Top and Heavy quarks crOss section calculatoR, Comput. Phys. Commun. 182 (2011) 1034 [arXiv:1007.1327] [InSPIRE].

[15] M. Beneke, P. Falgari, S. Klein and C. Schwinn, Hadronic top-quark pair production with NNLL threshold resummation, Nucl. Phys. B 855 (2012) 695 [arXiv:1109.1536] [INSPIRE].

[16] M. Beneke et al., Inclusive top-pair production phenomenology with TOPIXS, JHEP 07 (2012) 194 [arXiv: 1206.2454] [INSPIRE].

[17] V. Ahrens, A. Ferroglia, M. Neubert, B.D. Pecjak and L.L. Yang, Precision predictions for the $t+\bar{t}$ production cross section at hadron colliders, Phys. Lett. B 703 (2011) 135 [arXiv:1105.5824] [INSPIRE].

[18] M. Krämer et al., Supersymmetry production cross sections in pp collisions at $\sqrt{s}=7 \mathrm{TeV}$, arXiv: 1206.2892 [INSPIRE].

[19] K. Agashe, H. Davoudiasl, G. Perez and A. Soni, Warped gravitons at the LHC and beyond, Phys. Rev. D 76 (2007) 036006 [hep-ph/0701186] [INSPIRE].

[20] L. Randall and R. Sundrum, An alternative to compactification, Phys. Rev. Lett. 83 (1999) 4690 [hep-th/9906064] [INSPIRE].

[21] G.F. Giudice, R. Rattazzi and J.D. Wells, Graviscalars from higher dimensional metrics and curvature Higgs mixing, Nucl. Phys. B 595 (2001) 250 [hep-ph/0002178] [INSPIRE].

[22] R. Frederix and F. Maltoni, Top pair invariant mass distribution: a window on new physics, JHEP 01 (2009) 047 [arXiv:0712.2355] [INSPIRE].

[23] V. Barger, T. Han and D.G. Walker, Top quark pairs at high invariant mass: a model-independent discriminator of new physics at the LHC, Phys. Rev. Lett. 100 (2008) 031801 [hep-ph/0612016] [INSPIRE].

[24] P. Chiappetta and M. Perrottet, Possible bounds on compositeness from inclusive one jet production in large hadron colliders, Phys. Lett. B 253 (1991) 489 [INSPIRE].

[25] CMS collaboration, Search for contact interactions using the inclusive jet $p_{T}$ spectrum in pp collisions at $\sqrt{s}=7 \mathrm{TeV}$, Phys. Rev. D 87 (2013) 052017 [arXiv:1301.5023] [INSPIRE].

[26] CMS collaboration, Search for quark compositeness in dijet angular distributions from pp collisions at $\sqrt{s}=7 \mathrm{TeV}$, JHEP 05 (2012) 055 [arXiv: 1202.5535] [INSPIRE].

[27] ATLAS collaboration, ATLAS search for new phenomena in dijet mass and angular distributions using pp collisions at $\sqrt{s}=7$ TeV, JHEP 01 (2013) 029 [arXiv:1210.1718] [INSPIRE].

[28] D0 collaboration, V. Abazov et al., Measurement of the inclusive jet cross-section in $p \bar{p}$ collisions at $\sqrt{s}=1.96 \mathrm{TeV}$, Phys. Rev. Lett. 101 (2008) 062001 [arXiv:0802.2400] [INSPIRE].

[29] CDF collaboration, T. Aaltonen et al., Measurement of the inclusive jet cross section at the Fermilab Tevatron p $\bar{p}$ collider using a cone-based jet algorithm, Phys. Rev. D 78 (2008) 052006 [Erratum ibid. D 79 (2009) 119902] [arXiv:0807.2204] [INSPIRE]. 
[30] CMS collaboration, Measurements of differential jet cross sections in proton-proton collisions at $\sqrt{s}=7 \mathrm{TeV}$ with the CMS detector, Phys. Rev. D 87 (2013) 112002 [arXiv: 1212.6660] [INSPIRE].

[31] ATLAS collaboration, Measurement of inclusive jet and dijet production in pp collisions at $\sqrt{s}=7$ TeV using the ATLAS detector, Phys. Rev. D 86 (2012) 014022 [arXiv:1112.6297] [INSPIRE].

[32] D. d'Enterria and J. Rojo, Quantitative constraints on the gluon distribution function in the proton from collider isolated-photon data, Nucl. Phys. B 860 (2012) 311 [arXiv:1202.1762] [INSPIRE].

[33] L. Carminati et al., Sensitivity of the LHC isolated-gamma+jet data to the parton distribution functions of the proton, EPL 101 (2013) 61002 [arXiv:1212.5511] [INSPIRE].

[34] A. G.-D. Ridder, T. Gehrmann, E. Glover and J. Pires, Second order QCD corrections to jet production at hadron colliders: the all-gluon contribution, Phys. Rev. Lett. 110 (2013) 162003 [arXiv:1301.7310] [inSPIRE].

[35] R.D. Ball et al., A first unbiased global NLO determination of parton distributions and their uncertainties, Nucl. Phys. B 838 (2010) 136 [arXiv: 1002.4407] [INSPIRE].

[36] M. Czakon and A. Mitov, Top++: a program for the calculation of the top-pair cross-section at hadron colliders, arXiv:1112.5675 [INSPIRE].

[37] S. Alekhin, J. Blumlein and S. Moch, Parton distribution functions and benchmark cross sections at NNLO, Phys. Rev. D 86 (2012) 054009 [arXiv:1202.2281] [INSPIRE].

[38] J. Gao et al., The CT10 NNLO global analysis of QCD, arXiv:1302.6246 [INSPIRE].

[39] ZEUS, H1 collaboration, A. Cooper-Sarkar, PDF Fits at HERA, PoS (EPS-HEP2011) 320 [arXiv: 1112.2107] [INSPIRE].

[40] P. Jimenez-Delgado and E. Reya, Dynamical NNLO parton distributions, Phys. Rev. D 79 (2009) 074023 [arXiv:0810.4274] [INSPIRE].

[41] A. Martin, W. Stirling, R. Thorne and G. Watt, Parton distributions for the LHC, Eur. Phys. J. C 63 (2009) 189 [arXiv:0901.0002] [INSPIRE].

[42] R.D. Ball et al., Parton distributions with LHC data, Nucl. Phys. B 867 (2013) 244 [arXiv: 1207.1303] [INSPIRE].

[43] R.D. Ball et al., Parton distribution benchmarking with LHC data, JHEP 04 (2013) 125 [arXiv:1211.5142] [INSPIRE].

[44] P.M. Nadolsky and Z. Sullivan, PDF uncertainties in WH production at Tevatron, eConf C 010630 (2001) P510 [hep-ph/0110378] [INSPIRE].

[45] Particle Data Group collaboration, J. Beringer et al., Review of particle physics, Phys. Rev. D 86 (2012) 010001 [inSPIRE].

[46] H.-L. Lai et al., Uncertainty induced by QCD coupling in the CTEQ global analysis of parton distributions, Phys. Rev. D 82 (2010) 054021 [arXiv: 1004.4624] [INSPIRE].

[47] F. Demartin, S. Forte, E. Mariani, J. Rojo and A. Vicini, The impact of PDF and alphas uncertainties on Higgs Production in gluon fusion at hadron colliders, Phys. Rev. D 82 (2010) 014002 [arXiv:1004.0962] [INSPIRE].

[48] M. Whalley, D. Bourilkov and R. Group, The Les Houches accord PDFs (LHAPDF) and LHAGLUE, hep-ph/0508110 [INSPIRE]. 
[49] CDF, D0 collaboration, T. Aaltonen et al., Combination of the top-quark mass measurements from the Tevatron collider, Phys. Rev. D 86 (2012) 092003 [arXiv:1207.1069] [INSPIRE].

[50] LhC Higgs Cross Section Working Group collaboration, S. Dittmaier et al., Handbook of LHC Higgs Cross Sections: 1. Inclusive observables, arXiv:1101.0593 [INSPIRE].

[51] Tevatron electroweak working group, T. Aaltonen et al., Combination of the $t t$ production cross section measurements from the Tevatron collider, D0 Note 6363 (2012).

[52] ATLAS collaboration, Statistical combination of top quark pair production cross-section measurements using dilepton, single-lepton and all-hadronic final states at $\sqrt{s}=7$ TeV with the ATLAS detector, ATLAS-CONF-2012-024 (2012).

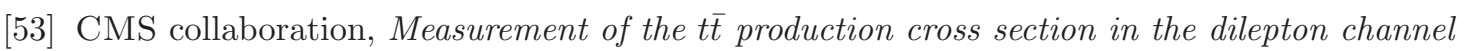
in pp collisions at $\sqrt{s}=7$ TeV, JHEP 11 (2012) 067 [arXiv:1208.2671] [INSPIRE].

[54] ATLAS collaboration, Measurement of the top quark pair production cross section in the single-lepton channel with ATLAS in proton-proton collisions at $8 \mathrm{TeV}$ using kinematic fits with b-tagging, ATLAS-CONF-2012-149 (2012).

[55] CMS collaboration, Combination of ATLAS and CMS top-quark pair cross section measurements using proton-proton collisions at $\sqrt{s}=7$ TeV, CMS-PAS-TOP-12-003 (2012).

[56] CMS collaboration, Top pair cross section in e/ $\mu+$ jets at $8 \mathrm{TeV}$, CMS-PAS-TOP-12-006 (2012).

[57] ATLAS and CMS collaboration, Combination of ATLAS and CMS top-quark pair cross section measurements using proton-proton collisions at $\sqrt{s}=7$ TeV, CMS-PAS-TOP-12-003 (2012).

[58] S. Forte, E. Laenen, P. Nason and J. Rojo, Heavy quarks in deep-inelastic scattering, Nucl. Phys. B 834 (2010) 116 [arXiv:1001.2312] [InSPIRE].

[59] R. Thorne, A variable-flavor number scheme for NNLO, Phys. Rev. D 73 (2006) 054019 [hep-ph/0601245] [INSPIRE].

[60] M. Guzzi, P.M. Nadolsky, H.-L. Lai and C.-P. Yuan, General-mass treatment for deep inelastic scattering at two-loop accuracy, Phys. Rev. D 86 (2012) 053005 [arXiv:1108.5112] [INSPIRE].

[61] R. Thorne, Effect of changes of variable flavor number scheme on parton distribution functions and predicted cross sections, Phys. Rev. D 86 (2012) 074017 [arXiv:1201.6180] [INSPIRE].

[62] NNPDF collaboration, R.D. Ball et al., Theoretical issues in PDF determination and associated uncertainties, Phys. Lett. B 723 (2013) 330 [arXiv:1303.1189] [INSPIRE].

[63] ATLAS collaboration, Determination of the top-quark mass from the $\bar{t} \bar{t}$ cross section measurement in pp collisions at $\sqrt{s}=7 \mathrm{TeV}$ with the ATLAS detector, ATLAS-CONF-2011-054 (2011).

[64] CMS colaboration, Determination of the top quark mass from the $t \bar{t}$ cross section at $\sqrt{s}=7 \mathrm{TeV}$, CMS-PAS-TOP-11-008 (2011).

[65] U. Langenfeld, S. Moch and P. Uwer, Measuring the running top-quark mass, Phys. Rev. D 80 (2009) 054009 [arXiv:0906.5273] [INSPIRE]. 
[66] D0 collaboration, V. Abazov et al., Determination of the strong coupling constant from the inclusive jet cross section in $p \bar{p}$ collisions at $\sqrt{s}=1.96 \mathrm{TeV}$,

Phys. Rev. D 80 (2009) 111107 [arXiv:0911.2710] [InSPIRE].

[67] B. Malaescu and P. Starovoitov, Evaluation of the strong coupling constant $\alpha_{s}$ using the ATLAS inclusive jet cross-section data, Eur. Phys. J. C 72 (2012) 2041 [arXiv:1203.5416] [INSPIRE].

[68] CMS collaboration, Measurement of the ratio of the inclusive 3-jet to 2-jet cross-sections in pp collisions at 7 tev and first determination of the strong coupling at transverse momenta in the TeV range, CMS-PAS-QCD-11-003 (2011).

[69] CMS collaboration, First determination of the strong coupling constant from the $t \bar{t}$ cross section, CMS-PAS-TOP-12-022 (2012).

[70] P.M. Nadolsky et al., Implications of CTEQ global analysis for collider observables, Phys. Rev. D 78 (2008) 013004 [arXiv:0802.0007] [InSPIRE].

[71] NNPDF collaboration, R.D. Ball et al., Reweighting and unweighting of parton distributions and the LHC W lepton asymmetry data, Nucl. Phys. B 855 (2012) 608 [arXiv:1108.1758] [INSPIRE].

[72] NNPDF collaboration, R.D. Ball et al., Reweighting NNPDFs: the W lepton asymmetry, Nucl. Phys. B 849 (2011) 112 [Erratum ibid. B 854 (2012) 926-927] [arXiv:1012.0836] [INSPIRE].

[73] T. Carli et al., A posteriori inclusion of parton density functions in NLO QCD final-state calculations at hadron colliders: The APPLGRID Project, Eur. Phys. J. C 66 (2010) 503 [arXiv:0911.2985] [INSPIRE].

[74] G. Watt and R. Thorne, Study of Monte Carlo approach to experimental uncertainty propagation with MSTW 2008 PDFs, JHEP 08 (2012) 052 [arXiv: 1205.4024] [INSPIRE].

[75] ATLAS collaboration, Measurements of top quark pair relative differential cross-sections with ATLAS in pp collisions at $\sqrt{s}=7$ TeV, Eur. Phys. J. C 73 (2013) 2261 [arXiv: 1207.5644] [INSPIRE].

[76] CMS collaboration, Measurement of differential top-quark pair production cross sections in pp colisions at $\sqrt{s}=7$ TeV, Eur. Phys. J. C 73 (2013) 2339 [arXiv:1211.2220] [InSPIRE].

[77] V. Ahrens, A. Ferroglia, M. Neubert, B.D. Pecjak and L.-L. Yang, RG-improved single-particle inclusive cross sections and forward-backward asymmetry in $\bar{t} \bar{t}$ production at hadron colliders, JHEP 09 (2011) 070 [arXiv: 1103.0550] [INSPIRE].

[78] R.D. Ball et al., Impact of heavy quark masses on parton distributions and LHC phenomenology, Nucl. Phys. B 849 (2011) 296 [arXiv:1101.1300] [INSPIRE].

[79] NNPDF collaboration, R.D. Ball et al., Unbiased global determination of parton distributions and their uncertainties at NNLO and at LO, Nucl. Phys. B 855 (2012) 153 [arXiv:1107.2652] [INSPIRE].

[80] J. Alwall et al., MadGraph/MadEvent v4: the new web generation, JHEP 09 (2007) 028 [arXiv: 0706 . 2334] [INSPIRE].

[81] CMS collaboration, Search for resonant $t \bar{t}$ production in lepton + jets events in pp collisions at $\sqrt{s}=7 \mathrm{TeV}$, JHEP 12 (2012) 015 [arXiv:1209.4397] [INSPIRE]. 
[82] ATLAS collaboration, A search for $t \bar{t}$ resonances in lepton+jets events with highly boosted top quarks collected in pp collisions at $\sqrt{s}=7 \mathrm{TeV}$ with the ATLAS detector, JHEP 09 (2012) 041 [arXiv: 1207.2409] [INSPIRE].

[83] ATLAS collaboration, A search for $t \bar{t}$ resonances with the ATLAS detector in $2.05 \mathrm{fb}^{-1}$ of proton-proton collisions at $\sqrt{s}=7$ TeV, Eur. Phys. J. C 72 (2012) 2083 [arXiv: 1205.5371] [INSPIRE].

[84] ATLAS collaboration, Search for resonances decaying into top-quark pairs using fully hadronic decays in pp collisions with ATLAS at $\sqrt{s}=7$ TeV, JHEP 01 (2013) 116 [arXiv: 1211.2202] [INSPIRE].

[85] R. Frederix et al., Four-lepton production at hadron colliders: aMC@NLO predictions with theoretical uncertainties, JHEP 02 (2012) 099 [arXiv:1110.4738] [INSPIRE].

[86] S. Frixione and B.R. Webber, Matching NLO QCD computations and parton shower simulations, JHEP 06 (2002) 029 [hep-ph/0204244] [INSPIRE].

[87] R. Frederix, S. Frixione, F. Maltoni and T. Stelzer, Automation of next-to-leading order computations in QCD: the FKS subtraction, JHEP 10 (2009) 003 [arXiv:0908.4272] [INSPIRE].

[88] G. Corcella et al., HERWIG 6: an event generator for hadron emission reactions with interfering gluons (including supersymmetric processes), JHEP 01 (2001) 010 [hep-ph/0011363] [INSPIRE].

[89] M.L. Mangano and J. Rojo, Cross section ratios between different CM energies at the LHC: opportunities for precision measurements and BSM sensitivity, JHEP 08 (2012) 010 [arXiv: 1206.3557] [INSPIRE].

[90] CMS collaboration, Search for pair produced fourth-generation up-type quarks in pp collisions at $\sqrt{s}=7 \mathrm{TeV}$ with a lepton in the final state, Phys. Lett. B 718 (2012) 307 [arXiv: 1209.0471] [INSPIRE].

[91] ATLAS collaboration, Search for pair production of heavy top-like quarks decaying to a high-p $T W$ boson and a $b$ quark in the lepton plus jets final state at $\sqrt{s}=7 \mathrm{TeV}$ with the ATLAS detector, Phys. Lett. B $\mathbf{7 1 8}$ (2013) 1284 [arXiv:1210.5468] [INSPIRE].

[92] CMS collaboration, Combined search for the quarks of a sequential fourth generation, Phys. Rev. D 86 (2012) 112003 [arXiv:1209.1062] [INSPIRE]. 